\title{
Neurobiological and behavioral mechanisms of circadian rhythm disruption in bipolar disorder: A critical multi- disciplinary literature review and agenda for future research from the ISBD task force on chronobiology
}

\author{
Michael J. McCarthy ${ }^{1,2}$ | John F. Gottlieb ${ }^{3}$ (D) | Robert Gonzalez ${ }^{4}$ | \\ Colleen A. McClung ${ }^{5}$ ( ) | Lauren B. Alloy ${ }^{6}$ | Sean Cain ${ }^{7}$ | Davide Dulcis ${ }^{1}$ | \\ Bruno Etain ${ }^{8} \odot$ | Benicio N. Frey ${ }^{9}$ | Corrado Garbazza ${ }^{10}$ | Kyle D. Ketchesin ${ }^{5}$ | \\ Dominic Landgraf $^{11}$ @ | Heon-Jeong Lee ${ }^{12}$ | Cynthia Marie-Claire ${ }^{8}$ | Robin Nusslock $^{13}$ | \\ Alessandra Porcu $^{1}$ | Richard Porter ${ }^{14} \odot$ | Philipp Ritter ${ }^{15}$ | Jan Scott ${ }^{16}$ Ji $_{\text {| }}$ \\ Daniel Smith ${ }^{17}$ | Holly A. Swartz ${ }^{5}$ (] | Greg Murray ${ }^{18}$ \\ ${ }^{1}$ UC San Diego Department of Psychiatry \& Center for Circadian Biology, La Jolla, California, USA \\ ${ }^{2}$ VA San Diego Healthcare System, San Diego, California, USA \\ ${ }^{3}$ Department of Psychiatry, Feinberg School of Medicine, Northwestern University, Chicago, Illinois, USA \\ ${ }^{4}$ Department of Psychiatry and Behavioral Health, Pennsylvania State University, Hershey, Pennsylvania, USA \\ ${ }^{5}$ Department of Psychiatry, University of Pittsburgh, Pittsburgh, Pennsylvania, USA \\ ${ }^{6}$ Department of Psychology, Temple University, Philadelphia, Pennsylvania, USA \\ ${ }^{7}$ School of Psychological Sciences and Turner Institute for Brain and Mental Health, Monash University, Melbourne, Victoria, Australia \\ ${ }^{8}$ Université de Paris, INSERM UMR-S 1144, Paris, France \\ ${ }^{9}$ Department Psychiatry and Behavioral Neuroscience, McMaster University, Hamilton, Ontario, Canada \\ ${ }^{10}$ Centre for Chronobiology, Psychiatric Hospital of the University of Basel and Transfaculty Research Platform Molecular and Cognitive Neurosciences, \\ University of Basel, Basel, Switzerland \\ ${ }^{11}$ Circadian Biology Group, Department of Molecular Neurobiology, Clinic of Psychiatry and Psychotherapy, University Hospital, Ludwig Maximilian University, \\ Munich, Germany \\ ${ }^{12}$ Department of Psychiatry and Chronobiology Institute, Korea University, Seoul, South Korea \\ ${ }^{13}$ Department of Psychology and Institute for Policy Research, Northwestern University, Chicago, Illinois, USA \\ ${ }^{14}$ University of Otago, Christchurch, New Zealand \\ ${ }^{15}$ Clinic for Psychiatry and Psychotherapy, Carl Gustav Carus University Hospital and Technical University of Dresden, Dresden, Germany \\ ${ }^{16}$ Institute of Neuroscience, Newcastle University, Newcastle, UK \\ ${ }^{17}$ Division of Psychiatry, University of Edinburgh, Edinburgh, UK \\ ${ }^{18}$ Centre for Mental Health, Swinburne University of Technology, Melbourne, Victoria, Australia
}

\section{Correspondence}

Greg Murray, Centre for Mental Health, Swinburne University of Technology,

Melbourne, Vic., Australia.

Email: gwm@swin.edu.au

\section{Abstract}

Aim: Symptoms of bipolar disorder (BD) include changes in mood, activity, energy, sleep, and appetite. Since many of these processes are regulated by circadian function, 
Funding information

VA Merit Award, Grant/Award Number:

BX003431; NCCIH, Grant/Award

Number: K99 AT010903; Kavli Institute

for Brain and Mind; German Research

Foundation; Emmy Noether Fellowship,

Grant/Award Number: LA4126/1-1;

NIMH, Grant/Award Number: MH077908,

$\mathrm{MH} 102310$ and $\mathrm{MH} 126911$ circadian rhythm disturbance has been examined as a biological feature underlying BD. The International Society for Bipolar Disorders Chronobiology Task Force (CTF) was commissioned to review evidence for neurobiological and behavioral mechanisms pertinent to BD.

Method: Drawing upon expertise in animal models, biomarkers, physiology, and behavior, CTF analyzed the relevant cross-disciplinary literature to precisely frame the discussion around circadian rhythm disruption in BD, highlight key findings, and for the first time integrate findings across levels of analysis to develop an internally consistent, coherent theoretical framework.

Results: Evidence from multiple sources implicates the circadian system in mood regulation, with corresponding associations with BD diagnoses and mood-related traits reported across genetic, cellular, physiological, and behavioral domains. However, circadian disruption does not appear to be specific to BD and is present across a variety of high-risk, prodromal, and syndromic psychiatric disorders. Substantial variability and ambiguity among the definitions, concepts and assumptions underlying the research have limited replication and the emergence of consensus findings.

Conclusions: Future research in circadian rhythms and its role in BD is warranted. Well-powered studies that carefully define associations between BD-related and chronobiologically-related constructs, and integrate across levels of analysis will be most illuminating.

\section{KEYWORDS}

actigraphy, animal models, biomarker, chronobiology, circadian, clock gene, levels of analysis, light, lithium, sleep

\section{1 | INTRODUCTION}

Involvement of disrupted circadian rhythms has been proposed across numerous dimensions of bipolar disorder (BD). This literature review from the International Society for Bipolar Disorders Chronobiology Task Force (CTF) is the final in a series, ${ }^{1,2}$ and specifically aims to critically evaluate the evidence supporting involvement of circadian rhythms in the neurobiology of BD. There is considerable interest in circadian rhythms in $\mathrm{BD}$, and the potential to develop diagnostic markers and treatments based on chronobiological principles. ${ }^{3}$ Already, therapeutic interventions based on circadian principles are included in contemporary professional treatment guidelines for BD. ${ }^{4}$ However, the ambiguous and incomplete chronobiology of BD literature limits utilization of these treatments, the development of standard protocols and the means to identify optimally responsive BD patients. Therefore, a more detailed characterization of the chronobiological underpinnings of $\mathrm{BD}$ is urgently required. The present review addresses this need by examining evidence across biobehavioral levels and outlining a program for future research. This project is innovative in its aim to review multidisciplinary research findings across animal models, human biomarkers, physiology, and behavior. Based upon this comprehensive and critical synthesis of evidence, the review provides novel commentary and agenda for future research.

\section{2 | METHODS}

The circadian system is unusual, if not unique, as a biobehavioral system in that its functions can be investigated with high fidelity and mechanistic detail across multiple levels of analysis starting in the genome and into cells, organs, physiological systems, and behavior. Because of this, chronobiological research in $\mathrm{BD}$ requires multidisciplinary integration. To achieve this integration as the basis for a rigorous critical review of relevant literature, MM and GM firstly developed a preliminary classification of the literature based on methods, namely, animal models, genetics, human biomarkers, and behavioral research. It was assumed that the findings from studies in these methodological domains would be largely incomparable due to differences in approach, outcome variables and conceptual assumptions. Given the disparate kinds of information collected across studies within domains, it was not possible to conduct a structured literature review at the domain level, or at the level of synthesis across domains. CTF planning meetings discussed levels of evidence of the review, and based on expert consensus it was agreed that strict levels of evidence hierarchies used in other CTF projects (e.g., PRISMA ${ }^{5}$ ) were not feasible. Workgroups were instructed to attend to quality of evidence, and to identify gaps or lack of replication across studies. To manage ambiguity, we used an inclusive and broad definition of the BD phenotype, accepted all measures and proxies of 
circadian function, and considered all types of associations whether implicit or explicitly defined in the identified literature.

Members of the CTF self-selected to join one of four workgroups to address each of these methodological domains. Each workgroup elected a Chair and developed three to four falsifiable propositions to focus the review within a domain (Table 1). Individual workgroup members were allocated to review the literature and draft text for each proposition. In most cases, individual members of workgroups had specific topic expertise in relation to the propositions (including having recently generated systematic and/or critical review of the literature). Each workgroup convened on three to four occasions during 2020 to discuss progress on the individual tasks. Activities of the four workgroups were coordinated by MM and GM, who were in communication throughout. To harmonize communication across groups, MM and GM played the role of Chair (Human Biomarkers and Behavior, respectively) or liaison (Physiology, Animal Models, respectively) for each workgroup.

Draft reviews from each workgroup were received in late 2020 and integrated by MM and GM to highlight consistent/contradictory findings and areas where additional evidence was required. Additional input from the CTF was incorporated to ensure accuracy and completeness of the integrated reporting and critical synthesis. All members of the CTF reviewed and approved the final version.

\section{3 | RESULTS}

The review addresses the pertinent neurobiology of circadian clocks (Section 3.1), then covers five multidisciplinary research themes that arose from the coordinated efforts of the CTF: Predisposing risk factors including vulnerability, heritability and genetic mechanisms (Section 3.2); Circadian disruption as a basis for defining sub-groups of BD (Section 3.3); Environmental risk factors, particularly light (Section 3.4); Specificity of circadian disruption in BD and across psychiatric disorders (Section 3.5); Circadian disruption across the illness course of BD (Section 3.6). Section 3.7 contains a tabular summary of key results from each of the subsections as a basis for the integrative critical Discussion (Section 4).

\subsection{Neurobiology of circadian clocks in BD}

In mammals, the suprachiasmatic nucleus (SCN) in the anterior hypothalamus is the master circadian pacemaker and is important for organizing daily $24 \mathrm{~h}$ rhythms in sleep/wake, activity, and other physiological processes and behaviors. Nearly all cells in the body express the circadian clock genes that support circadian rhythms, and many can sustain autonomous rhythms in isolated experimental preparations such as tissue slices and cell cultures (see Box 1). Included in these tissues are brain regions such as frontal cortex, limbic regions, ventral tegmentum and ventral striatum that are implicated in reward pathways, affect and BD. In a study of baboons (a diurnal, non-human primate), gene expression was measured using RNA sequencing across the genome at 12 time points across 64 distinct tissues, including 22 brain regions. ${ }^{6}$ Most brain regions examined showed rhythmic profiles, indicating the presence of cellular rhythms. However, each region was distinct both in the number and ensembles of rhythmic genes demonstrating context-dependence. Notably, rhythmic expression profiles were more likely to be found in genes serving essential processes in each tissue. These data indicate that while the $\mathrm{SCN}$ serves a critical role coordinating rhythms across the body, circadian rhythms are widespread across the nervous system, and interface with numerous processes that underlie healthy brain function and pathological processes that potentially contribute

TAB LE 1 Workgroups and propositions to methods-based reviews

\begin{tabular}{|c|c|c|}
\hline Methods & Personnel & Propositions \\
\hline Animal models & 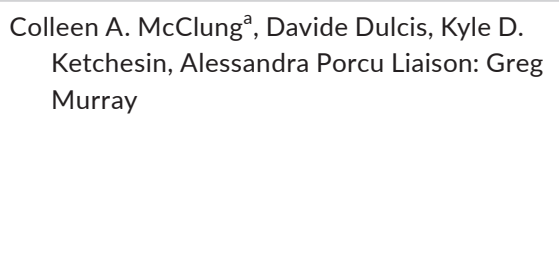 & $\begin{array}{l}\text { 1. SCN rhythms are essential for affect regulation } \\
\text { 2. Animal mutants are useful models for clock gene variation and effects } \\
\text { on mood in humans } \\
\text { 3. Experimental manipulation of light in animal models affects brain } \\
\text { pathways for mood regulation } \\
\text { 4. Mood stabilizing drugs engage the molecular clock as a therapeutic } \\
\text { mechanism }\end{array}$ \\
\hline Physiology & $\begin{array}{l}\text { Robert Gonzalez }{ }^{a} \text {, Sean Cain, Benicio Frey, } \\
\text { Corrado Garbazza, John Gottlieb, Philip } \\
\text { Ritter Liaison: Michael McCarthy }\end{array}$ & $\begin{array}{l}\text { 1. Light exposure plays a causal role to initiate } \mathrm{BD} \\
\text { 2. Light exposure plays a role in the phenotypic expression of BD } \\
\text { 3. Melatonin is fundamental to pathophysiology of BD } \\
\text { 4. Melatonin plays a role in phenotypic expression of BD }\end{array}$ \\
\hline $\begin{array}{l}\text { Human } \\
\text { biomarkers }\end{array}$ & $\begin{array}{c}\text { Michael McCarthy }{ }^{a} \text {, Bruno Etain, Heon-Jeong } \\
\text { Lee, Cynthia Marie-Claire, Daniel Smith }\end{array}$ & $\begin{array}{l}\text { 1. Variation in clock genes is essential to the pathophysiology of BD } \\
\text { 2. Circadian abnormalities identify distinct BD subgroups } \\
\text { 3. Circadian rhythm abnormalities are specific to BD } \\
\text { 4. Mood episodes are associated with circadian misalignment }\end{array}$ \\
\hline Behavior & 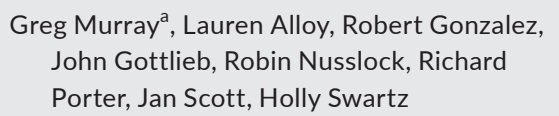 & $\begin{array}{l}\text { 1. Circadian abnormalities are measurable pre-onset (vulnerability) } \\
\text { 2. Circadian abnormalities are causal in symptoms and episodes } \\
\text { 3. Polarity of episodes are explained by phase delay/advance }\end{array}$ \\
\hline
\end{tabular}

Abbreviation: BD, bipolar disorder.

${ }^{a}$ Workgroup leader. 
to neuropsychiatric disorders like BD. Therefore, loss of rhythm in a particular brain region and/or loss of coordination among brain regions is predicted to have detrimental effects on brain function that would be observable as changes in behavior, cognition and affect.

\section{BOX 1 Definition of clock gene}

We define clock genes as those genes critically involved in the $24 \mathrm{~h}$ molecular timekeeping mechanism in mammalian cells. Clock genes are usually defined in rodents by loss of function mutations and subsequent effects on behavior observed under constant lighting conditions. With redundancy in the system, few clock gene mutants are fully arrhythmic under constant conditions, but many show irregular rhythm parameters and/or become arrhythmic with the loss of additional clock genes. There are 20 established clock genes (Figure 1). The list is frequently growing to include new genes as candidates that may require additional description and validation.

\subsection{1 | Molecular clock network}

Clock genes form a transcriptional/translational feedback loop to maintain the essential functions underlying cellular circadian rhythms (Figure 1). At the center of this loop, the proteins CLOCK and BMAL1 (encoded from ARNTL) bind to form a heterodimeric transcriptional activator. The CLOCK/BMAL1 complex binds to Ebox promoter elements in DNA, driving the expression of PER1/2/3 and $C R Y 1 / 2$, transcriptional repressors that inhibit their own expression over $\sim 24 \mathrm{~h}$ cycles to sustain a circadian oscillator. PER and CRY proteins coordinate to determine period length through negative feedback of CLOCK/BMAL1. In some instances, NPAS2 can substitute for CLOCK and may play an important role in cell types where NPAS2 expression is abundant, including medium spiny striatal neurons and other forebrain regions. ${ }^{7,8}$ Additional feedback loops have been described (e.g., REV-ERB $\alpha / \beta, R O R A / B / C, D E C 1$, and DBP) that modulate rhythms in tissue-specific contexts. In the SCN, calcium and associated signal transduction pathways serve as clock inputs, linking the retinohypothalamic tract to the circadian clock through post-translational modification of clock proteins and modulation of transcription factors that regulate clock genes like PER1/2 to entrain

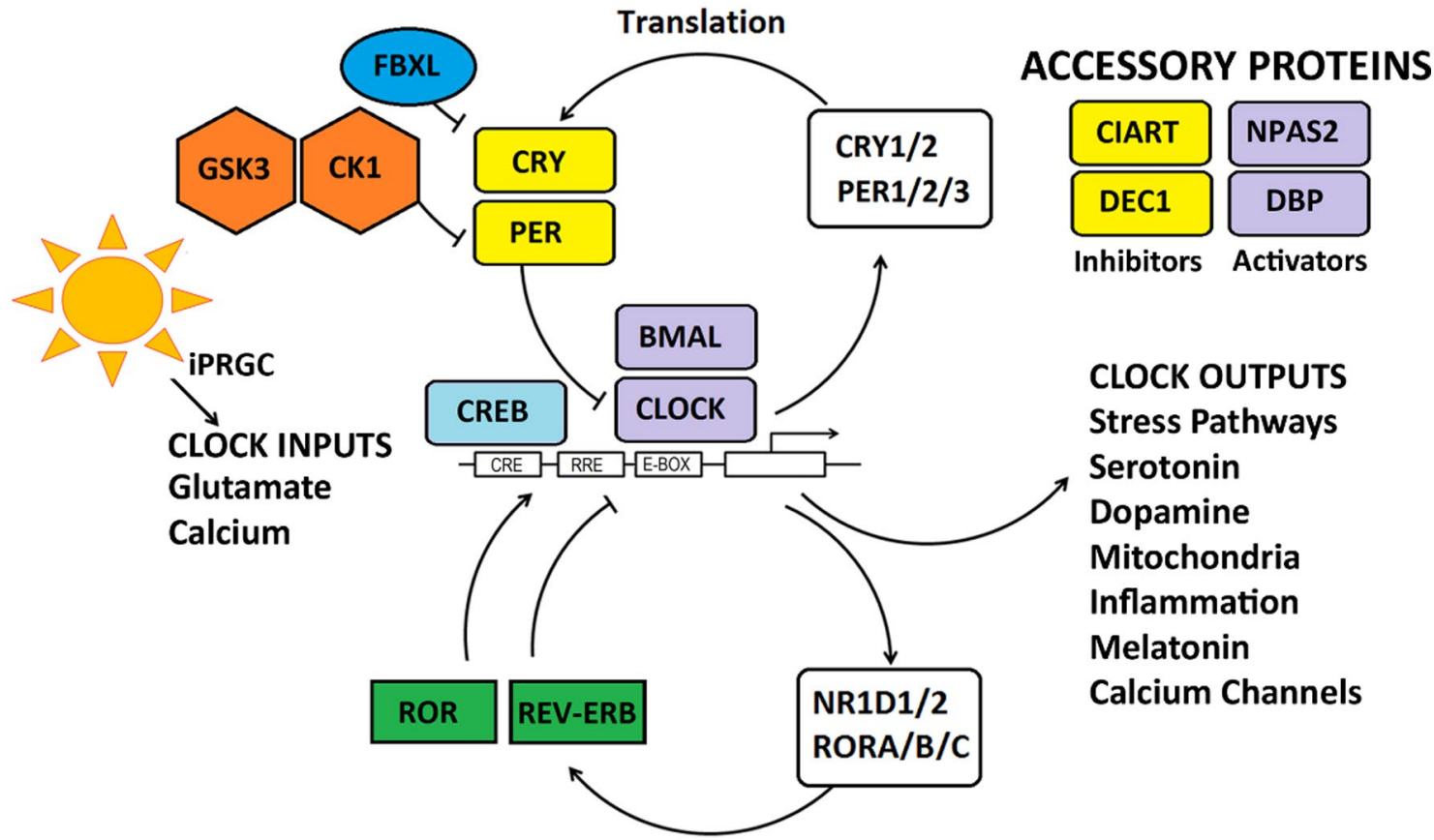

FIGURE 1 Molecular clock comprises clock genes, organized in transcriptional/translational feedback loops. Transcriptional activator proteins (purple), BMAL1 and CLOCK bind to DNA elements in promoters (E-Box) to stimulate transcription of CRY1/2, PER1/2/3, NR1D1/2, and RORA/B genes that encode transcriptional repressors (yellow) or secondary loops (green) to regulate activity through protein and DNA interactions. These interactions yield oscillatory changes in gene expression and translation over $24 \mathrm{~h}$ cycles causing cellular rhythms. Posttranslational modification by protein kinases (glycogen synthase 3 kinases, GSK3, casein 1 kinases, CK1) or ubiquitin ligases (FBXL) adjust the rate of protein turnover. Light and environmental inputs to intrinsically photosensitive retinal ganglion cells (ipRGC) communicate with the clock through glutamate, pituitary adenylate-cyclase-activating polypeptide (PACAP) and intracellular calcium to shift circadian phase through actions on CAMP-response element-binding protein (CREB). Accessory activators (NPAS2 and DBP) and inhibitors (CIART and DEC) play important roles in some cell types. The circadian clock affects the expression of clock-controlled genes that are rhythmically expressed, but do not play a role in the timekeeping mechanism. These rhythmic clock outputs are involved in physiological pathways that affect brain functions that require precise timing across the $24 \mathrm{~h}$ cycle 
rhythms to light. ${ }^{9}$ Post-translational modification of proteins by kinases (e.g., glycogen synthase 3 kinases [GSK3], and casein kinase 1 [CSNK1]), ubiquitin ligases (e.g., FBXL3) and other molecules also regulate turnover of proteins to affect temporal kinetics of the feedback loop. The clock interfaces with stress pathways. ${ }^{10}$ For instance, CHRONO (encoded from CIART) is upregulated by stress in the hypothalamus and is a transcriptional repressor of CLOCK/BMAL1. ${ }^{11,12}$

\subsection{2 | Role of the SCN in mood regulation}

Past research on the neurobiology of mood regulation in animal models of mood disorders has focused on the ventral striatum (nucleus accumbens), amygdala, hippocampus, cortical regions, and monoaminergic inputs from the ventral tegmentum (VTA), raphe nucleus and locus coeruleus. The circadian rhythm hypothesis of BD has also drawn attention to the SCN. Several rodent studies have manipulated the $\mathrm{SCN}$ to determine its role in mood-related behavior. Counterintuitively, lesions of the SCN result in less depression-like behavior in the forced swim test. ${ }^{13,14}$ However, these unexpected findings are difficult to interpret since pathways traversing the SCN were likely destroyed and a lack of behavioral assessment over circadian time scales. To determine the role of the BMAL1 in mood regulation in a tissue restricted and neuroanatomically intact system, Bmal1 short hairpin RNA (shRNA) was injected into the SCN of mice subsequently tested across a battery of behavioral assays. ${ }^{15}$ Bmal1 knockdown in the SCN resulted in increased depression-like and anxiety-like behaviors. Interestingly, SCN tissue explants were rendered arrhythmic but wheel-running rhythms remained intact. These results indicate that redundancies in the SCN may preserve circadian rhythms despite the disruption to overlapping affectreward systems. Therefore, preliminary anatomical data support the idea that the SCN is linked to brain reward circuits and contributes to affect regulation.

\subsection{3 | Photic inputs to the SCN}

Circadian clocks require external time signals (zeitgebers) to coordinate the internal biological activity of an individual with the environment. In humans, zeitgebers include temperature variation, and timing of meals, exercise, and social interaction. But in all mammals including humans, light is the most salient entrainment signal for the circadian clock. A class of melanopsin expressing, intrinsically photosensitive retinal ganglion cells (ipRGCs) are responsible for photic entrainment of clocks. ${ }^{16-18}$ Unlike other retinal photoreceptors, ipRGCs are primarily non-image-forming and have strong projections to the SCN, where they influence circadian rhythms. ${ }^{16}$ IpRGCs also have direct projects to other subcortical structures in the amygdala, hypothalamus, and diencephalon. By communicating light information to these brain regions, ipRGCs play a role regulating mood, learning and memory. ${ }^{17,19,20}$ In mice, it was shown that ipRGCs mediate light-induced mood changes through input to the perihabenular nucleus of the dorsal thalamus, and that ipRGCs were necessary and sufficient for effects of light on mood. ${ }^{19}$ The study concluded that the mood-related pathway is SCN-independent. However, a $7 \mathrm{~h}$ light/dark (LD) cycle was used to force the SCN rhythm to "free run" independently of photic entrainment. The non-physiological nature of this method leaves open the question of SCN involvement. In humans, light can suppress functional magnetic resonance image activity in the habenula ${ }^{21}$ and amygdala ${ }^{22}$ and increase connectivity between the ventromedial prefrontal cortex and amygdala. In these studies, light simultaneously reduced negative affect, and suggesting it may regulate emotional control through this mechanism. ${ }^{22}$

\subsubsection{Output mechanisms of the circadian clock}

BD presumably occurs from widespread dysfunction across neuronal systems, resulting in behavioral abnormalities manifesting primarily as mood and activity disturbances, with associated changes in arousal, cognition, and attention and brain structure. However, $\mathrm{BD}$ is also associated with endocrine abnormalities and changes in peripheral physiology. Clock-controlled genes (CCGs, Box 2) are rhythmically expressed in context-specific manner, often involved in the essential functions of a particular cell type and enriched in therapeutic drug targets. ${ }^{23}$ By way of CCGs acting in separate organ systems, disrupted circadian rhythms might affect a variety of cellular and physiological processes, possibly explaining the wide range of effects of BD on various dimensions of health. ${ }^{24-28}$ Key CCG outputs are reviewed below. The list is not meant to be exhaustive, but rather highlights key BD-associated processes regulated by CCGs that may conceivably be affected by disrupted circadian rhythms.

\subsection{5 | Melatonin}

Melatonin secretion is a key mechanism by which the central clock coordinates rhythms across the brain and peripheral organ systems. Twin studies reveal substantial heritability and variability in the levels of nocturnal melatonin secretion and its sensitivity to light. ${ }^{29}$ Several studies have reported that individuals with BD demonstrate a hypersensitivity to nocturnal light ${ }^{29-31}$ across a wide range of light intensities. $^{32}$ In one study comparing euthymic, medication-free BD participants and matched controls, a two-fold increase in melatonin suppression was observed in the BD group. ${ }^{31}$ Some studies suggest that the hypersensitivity to light, measured with melatonin suppression may be a trait marker of $\mathrm{BD} .{ }^{30,31}$ Acetylserotonin Omethyltransferase (ASMT) is a key enzyme involved in the biosynthesis of melatonin. Both rare mutations and a common polymorphism in ASMT linked with lower expression have been associated with BD in candidate case-control studies. ${ }^{33}$ Furthermore, in a study using actigraphy in 53 euthymic BD patients and 28 controls, subjects homozygous for a low activity ASMT allele showed association with longer sleep and interdaily stability. ${ }^{34}$ However, not all findings support a trait association with melatonin-suppression ${ }^{35-38}$ : One study 


\section{BOX 2 Clock controlled genes}

In genome-wide surveys, $50 \%-80 \%$ of genes were found to be expressed rhythmically in at least one cell type. However, these rhythms are narrowly expressed and the majority of these genes show limited rhythmicity in other tissues. Moreover, they differ from clock genes as they are not critically involved in maintaining molecular oscillators in cells. Instead, clock-controlled genes comprise output pathways of the circadian clock that exert circadian control over physiological processes for which daily timing is essential such as nutrient metabolism, cognition, goal directed activity, and immune function.

conducted in euthymic, medication free BD subjects ${ }^{37}$ and one conducted in BD subjects who were symptomatic and medicated ${ }^{38}$ failed to demonstrate hypersensitivity to light compared to controls. Similarly, differences in melatonin suppression did not appear in an investigation of monochromatic blue or red light under dark conditions. ${ }^{36}$ A recent study in major depressive disorder (MDD) supports the idea that melatonin suppression may change with state, showing that remitted MDD patients had nearly identical melatonin suppression responses to controls, whereas those currently depressed had reduced melatonin suppression. ${ }^{39}$ During episodes of mania, patients with $\mathrm{BD}$ have been shown to display elevated daytime levels of melatonin compared to depressive patients or control subjects. ${ }^{30,40}$ Studies of BD have also examined the effects of mood state on melatonin secretion but have not measured light sensitivity. In depressed subjects, a comparison of 14 patients with BD and 18 patients with MDD revealed significantly lower melatonin levels and later onset of secretion in the BD subjects. ${ }^{41}$ Further studies with clearly defined light exposure protocols, larger cohorts and longitudinal mood assessments are required to clarify the role of melatonin in BD.

\subsection{6 | Serotonin}

The human brain shows pronounced diurnal variation in serotonin (5HT) levels, a neurotransmitter strongly linked with mood disorders. ${ }^{42}$ Properties of the 5-HT system in mice including neuronal firing rate in the dorsal raphe nucleus, and brain neurotransmitter content are sensitive to light conditions and alteration of photoperiod. ${ }^{43}$ The rate limiting step in 5-HT synthesis is under the control of tryptophan hydroxylase, an enzyme that shows diurnal variation in in the raphe nuclei in response to signals from the SCN, endocrine, and behavioral cues. ${ }^{44,45}$

\subsection{7 | Dopamine}

Mania in BD is associated with increased dopamine activity. ${ }^{46,47}$ In the striatum, dopamine receptors, the dopamine transporter (DAT), and monoamine oxidase A (MAOA) all have strong diurnal rhythms in expression regulated by circadian proteins. ${ }^{48-50}$ Tyrosine hydroxylase, the rate limiting enzyme in dopamine synthesis shows diurnal rhythms in expression controlled by CLOCK and REV-ERB $\alpha$ proteins. ${ }^{51,52}$ Loss of function mutations of either protein leads to a hyperdopaminergic state and mania-like behavior in mice. ${ }^{51,53,54}$

\subsection{8 | Calcium}

Genome wide association studies (GWAS) of BD strongly implicate calcium channel genes such as CACNA1C. ${ }^{55}$ In neurons, rhythmic regulation of calcium signaling is important for neuronal activity and neuron/glial communication. In the SCN, intracellular $\mathrm{Ca}^{2+}$ levels have distinct rhythms that are coordinated with ATP accumulation and Per2 expression. ${ }^{56}$ Following a light pulse, Cacna1c null mutant mice have a reduced ability to phase advance circadian rhythms. ${ }^{57}$ In human fibroblasts, CACNA1C is rhythmically expressed, but BD patient cells carrying the CACNA1C risk allele have a reduction in this rhythm. ${ }^{58}$

\subsection{9 | Neuroinflammation}

Neuroimmune mechanisms may be involved in the pathophysiology of BD and can be connected to circadian rhythms in many ways. ${ }^{59-61}$ Circadian rhythm disruption can increase levels of inflammatory cytokines. ${ }^{62-64}$ CLOCK activation can increase NFKB mediated transcription, ${ }^{65,66}$ whereas inflammation can alter Per1 and Per2 expression in the hypothalamus and liver, ${ }^{67}$ and induce phase delays in the SCN. ${ }^{68,69}$ Inflammatory cytokines can disrupt spontaneous electrical activity rhythms in the SCN. ${ }^{70}$

\subsubsection{0 | Mitochondria}

The brain is vulnerable to oxidative stress and several studies have linked mitochondrial dysfunction to BD. ${ }^{71-73}$ Several mitochondrial functions have circadian rhythms. ${ }^{74}$ The molecular clock coordinates antioxidant response and mitochondrial functions through Sirtuin 1 (SIRT1) and nuclear factor erythroid-derived 2-like 2 (NRF2). SIRT1 becomes active in the presence of nicotinamide adenine dinucleotide $\left(\mathrm{NAD}^{+}\right.$) and binds to CLOCK/BMAL1 to regulate transcription. ${ }^{75,76}$ In brain astrocytes, NRF2 is regulated by CLOCK. Arrhythmic Clock mutant mice show reduced levels of NRF2 and signs of oxidative damage. $^{77,78}$

\subsubsection{1 | Sleep and circadian rhythms}

Sleep problems are commonly used as evidence of circadian disruption. Sleep has multiple components, some of which are regulated by CCGs, but others controlled by non-circadian factors. Contemporary 
conceptualizations of sleep focus on the two-factor model in which the non-circadian homeostatic sleep system interacts with the circadian pacemaker to determine overall sleep characteristics. ${ }^{79}$ In this way, sleep is strongly influenced by duration of wakefulness and an indirect marker of circadian rhythm (Box 3). The sleep parameters most strongly influenced by circadian function include phase and amplitude (i.e., sleep and activity consolidation). Sleep latency, staging, and overall quality, are less related to circadian rhythms. While many aspects of sleep may have important roles in $\mathrm{BD}$, sleep is considered primarily from the circadian perspective in the present literature review.

\subsection{Circadian disruption as a predisposing risk factor in BD}

\subsubsection{Vulnerability for BD}

High risk for BD can be defined in multiple different ways including familial risk, seprodromal cases (sub-threshold symptoms of mania/ hypomania), or temperamental features that may predispose an individual to a psychiatric disorder. Major findings from studies of these high-risk groups and circadian rhythm abnormalities are discussed in detail in following sections and summarized in Figure 2.

\section{Individuals at familial high risk for $B D$}

Based on familial clustering of mental illnesses, the offspring of BD parents are often considered to be at high risk for developing BD. In a study of young adults at high risk for BD based on symptoms and/or family history compared to individuals with BD and controls, sleep/activity profiles differed between BD cases and controls. Profiles of high-risk individuals were similar to full threshold BD cases showing both insomnia and hypersomnia, sensitivity to shifts in rhythms, difficulty awakening and prolonged sleep latency. ${ }^{80}$ In a study of 25 offspring of BD parents (OSBD) and 22 matched

\section{BOX 3 Circadian versus diurnal rhythms}

Biological rhythms entrain to environmental cues, especially light. Light effects may "mask" aberrant rhythms, and preserve rhythms aligned with the light/dark cycle, even in the context of a severely impaired circadian clock. For this reason, the term "circadian rhythm" should be reserved for rhythms observed under constant light or dark conditions. Most human and clinical studies have been performed under naturalistic day/wake-night/sleep conditions and not under controlled light/dark conditions in a laboratory. Therefore, while the term "circadian rhythm" is commonly used in the literature, it is more accurate to consider these patterns "diurnal rhythms", as their endogenous origin has not been confirmed. control offspring (OSC), group differences were noted in subjective sleep quality. ${ }^{81}$ However, these were not significant after adjusting for mood and anxiety symptoms. Social rhythms and actigraphy measures (Figure 2) did not differ between groups. ${ }^{81} \mathrm{~A}$ second study found similar results with nominally significant changes in sleep and greater eveningness in OSBD $(n=43)$ compared to OSC $(n=42)$ interpreted as decreased need for sleep in the OSBD group, but no differences measures of actigraphy, sleep onset/offset or daytime functioning. ${ }^{82}$ In 28 OSC, 13 OSBD, 23 OS of parents with MDD (OSMD), sleep duration was longest in OSMD. Sleep duration was significantly longer in OSBD versus OSC, but only over weekends and not during weekdays. ${ }^{83}$ In a study of depressed adolescents, OSBD had a significantly longer sleep duration and greater daytime dysfunction compared to $\mathrm{OSC}^{84}$ and were more likely to report hypersomnia, and increased appetite. ${ }^{85}$ With respect to sleep mechanisms of mood regulation, shorter sleep duration in adolescent OSBD was selectively associated with increased activity in brain reward circuits. ${ }^{86}$ Overall, family studies provide only limited evidence of circadian disruption in high-risk individuals. Quantitative rhythm measures using actigraphy or social rhythm metrics have failed to reveal BD-associated differences, whereas indirect links to circadian rhythms such as sleep abnormalities and greater eveningness have been observed in OSBD.

\section{High-risk community samples and BD spectrum cases}

Symptoms of hypomania in healthy individuals are considered risk factors for BD. Children at high-risk for BD based on hypomanic symptoms have been reported to have irregular timing of daytime activities and more variable sleep duration than controls, despite having similar average sleep duration. ${ }^{87} \mathrm{~A}$ later study noted that, compared to a matched low-risk group, actigraphy recordings in high-risk subjects showed significantly more delays and variability in sleep, more sleep fragmentation and lower relative amplitude. ${ }^{88}$ In a study of 313 community adults, hypomanic symptoms were not associated with chronotype, but irritability and risk-taking were associated with greater eveningness and irregular sleep-wake times. ${ }^{89}$ In contrast, depression was correlated with morningness in a study of 140 students. ${ }^{90}$ In other studies of high- and low-risk young adults, significantly lower amplitude activity rhythms were observed in the high-risk group, especially in individuals with a history of depression and high scores on a mania scale, but no group differences were found in social rhythms. ${ }^{91-93}$ Using the mood disorder questionnaire to stratify risk, lower amplitude activity rhythms were again seen in the high-risk group, but with no difference in chronotype between the high and low risk groups. ${ }^{94}$ In summary, low amplitude activity rhythms and delays are commonly reported across several actigraphy studies of high-risk groups defined by hypomanic symptoms. Other proxy measures of circadian rhythm such as chronotype, social rhythms have also been reported, but less consistently. Notably, the amplitude of activity rhythms may not reflect the amplitude of central circadian rhythms directed by the SCN and may be confounded by masking effects. Based on the identification of motor activity amplitude abnormalities in BD, further investigation 
(A)

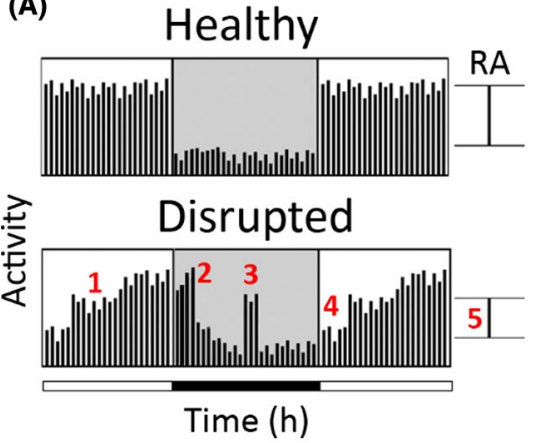

(C)
(B)
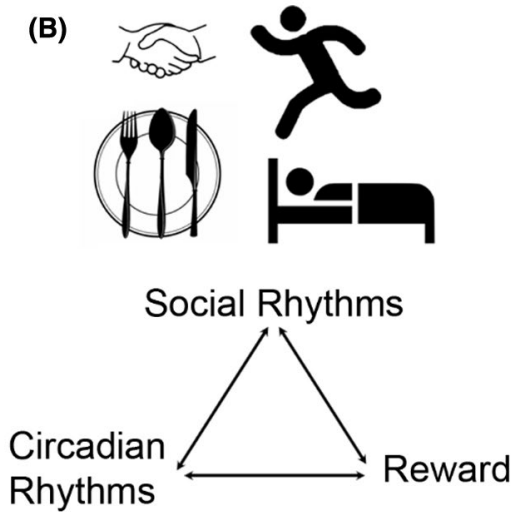

\begin{tabular}{|c|c|}
\hline High Risk Group & Findings \\
\hline Familial & Sleep: more variable \\
\hline Prodromal & $\begin{array}{l}\text { Activity: delays, frag } \\
\text { Rhythm: low relativ } \\
\text { Social rhythms: vari }\end{array}$ \\
\hline Hyperthymic $\longrightarrow$ & Morning Chronotyp \\
\hline $\begin{array}{l}\text { Cyclothymic/ - } \\
\text { Irritable }\end{array}$ & Evening Chrono \\
\hline
\end{tabular}

FIGURE 2 Actigraphy, social rhythm metrics, and schematic summary of emerging findings from ongoing studies of cohorts at high risk for bipolar disorder. (A) A hypothetical actigraphy study of participants with healthy and disrupted activity patterns, demonstrating some of the commonly reported findings in BD including (1) reduced activity, (2) delayed sleep onset, (3) fragmented sleep, (4) delayed activity onset, (5) low relative amplitude (RA). Black vertical bars indicate epochs of activity. Background color indicates observations during the day (white) and night (gray). Horizontal line indicates environmental light conditions of light (white) and dark (black). B) Behaviors including social interactions, work, exercise, meals and sleep may serve both as time cues and outputs of circadian rhythms that can be quantified using social rhythm metrics (top). Social rhythms, brain reward mechanisms and circadian rhythms interact. Abnormal findings in one of these may reflect disruption in one or more of the others (bottom). (C) Summary of major findings from studies of circadian rhythms in variously defined groups at high-risk for BD

into the relationship between motor activity and central rhythms is warranted.

BD exists on a spectrum that may include temperamental features, and hyperthymic temperament has been proposed as a risk factor for BD. In studies of healthy young adults, variability in sleep duration predicted scores of hyperthymia. ${ }^{95}$ Hyperthymic temperament has been associated with morning chronotype, while depressive, cyclothymic, irritable, and anxious temperaments were significantly associated with evening chronotype. ${ }^{96}$ A large-scale internet-based survey of chronotype and temperament in 5129 adults reported similar results: morningness was associated with hyperthymia, whereas eveningness was associated with cyclothymic, dysthymic, or disinhibited temperaments. ${ }^{97}$ In 186 adolescents, eveningness was associated with affective problems, attention difficulties, and rule-breaking. ${ }^{98}$ Overall, existing data indicate that hyperthymic temperament is associated with morning chronotype, while other affective temperaments and high-risk groups for BD show association with evening chronotype. This suggests hyperthymic temperament has distinct chronobiological characteristics compared to these other traits and the relationships among them relating to BD remain to be defined.

\subsubsection{Heritability of circadian rhythm phenotypes and BD}

\section{Genetic underpinnings of $B D$}

BD is heritable, with twin studies showing $60 \%-90 \%$ concordance for BD I, ${ }^{99}$ and GWAS showing that $20 \%-25 \%$ of variance is attributable to common genetic risk, with dozens of genetic risk factors identified for BD. ${ }^{55,100}$ Rare genetic variants have also been identified. ${ }^{101,102}$ Importantly, it is also clear from these studies that the genetic risk for BD overlaps with that of other psychiatric disorders such as schizophrenia ( $\mathrm{SCHZ}$ ) and MDD, with BD I more similar to the former and BD II more similar to the latter. ${ }^{55,100}$

\section{Family and twin studies}

Studies of high-risk cohorts indicate that circadian disruption may be a heritable risk factor for BD. Indeed, most of the sleep/wake patterns and chronotype markers associated with BD are also found in healthy relatives of patients and individuals with elevated risk of BD. ${ }^{103}$ Twin studies reveal that individual variation in daily activity is heritable. ${ }^{104}$ For example, in a family study of BD patients covering 26 pedigrees, including 136 BD and 422 unaffected relatives, shared heritability of rhythmic activity traits and BD was examined. ${ }^{105}$ 
Euthymic individuals with BD had longer sleep duration and later wake times compared to unaffected relatives. Moreover, the study identified $>40$ heritable phenotypes related to rest-activity timing in the BD pedigrees, with the strongest linkage for an actigraphyderived measure of intradaily variability, ${ }^{106}$ but also evidence of fragmentation and lower overall daytime activity. Importantly, many of the activity-associated loci overlapped with BD-associated loci. Similar results were found in BD subjects and their healthy relatives using smart-phone based activity measures. ${ }^{107}$ Compared to controls, BD subjects with mild-moderate depression had less activity and fewer social interactions, whereas euthymic unaffected relatives had decreased activity but typical social interactions suggesting activity is an intermediate phenotype.

\subsubsection{Genetic mechanisms in the molecular clock and risk for BD}

\section{Candidate gene studies in animals and humans}

Genetic studies using inbred animals are commonly helpful for demonstrating the effects of a given gene compared to human studies where genetic variation is substantially higher and single gene effects may be harder to detect. Clock genes have been systematically mutated in mice to determine their role in affective behaviors. ${ }^{108}$ Importantly, clock genes are conserved in humans. Accordingly, most have also been examined in candidate gene association studies in BD using single nucleotide polymorphisms (SNP). By modern standards, the candidate gene studies are considered underpowered. Nonetheless, some have yielded findings that were subsequently supported by GWAS. ${ }^{109}$ Many of these studies have examined multiple aspects of the BD phenotype, making replication of these studies particularly problematic and prone to false positive association.

\section{CLOCK}

One of the best characterized animal models of BD is the Clock $\Delta 19$-mutant mouse. Clock $\Delta 19$ mice encode a dominantnegative CLOCK protein, causing reduced amplitude, long period, delayed phase under a light/dark cycle and arrhythmic behavior under constant darkness. ${ }^{110}$ Clock $\Delta 19$ mice display a mania-like phenotype consisting of increased dopamine transmission, hyperactivity, increased reward-seeking and impulsivity-like behaviors, and decreases in depressive-like behavior, traits that are partly reversible by lithium. ${ }^{111-113}$ In humans, common variants in CLOCK have been associated with BD and related phenotypes in candidate gene association studies, ${ }^{114-117}$ but have not been consistently replicated. ${ }^{118}$

\section{ARNTL}

BMAL1 null mutant mice shows reduced lifespan and widespread physical abnormalities, ${ }^{119}$ limiting study of this mutant. Perhaps for this reason, it is also relatively understudied in human genetic studies. However, variants in ARNTL have been linked with $B D,{ }^{120,121}$ and an epigenetic study suggests methylation of ARNTL was associated with BD. ${ }^{122}$ BMAL1 null mutant macaque monkeys show irregular sleep/activity cycles, elevated stress hormones and increased psychosis, depression and anxiety-like behaviors. ${ }^{123}$

\section{PER1/2/3}

Per1 mutant mice show allele-specific phenotypes. Per1 ${ }^{\mathrm{Brdm}-/}$ mutants display reduced response to psychostimulants and decreased reward-seeking, particularly after experimentally induced stress, ${ }^{124-126}$ whereas Per1 ${ }^{\text {ldc-l- }}$ mutation does not cause an affective phenotype. ${ }^{127}$ Per2 $2^{\text {Brdm1-/- }}$ mutants display reduced immobility in the forced swim test, and increased sensitivity to cocaine and alcohol. ${ }^{124,128}$ Mutant mice lacking either Per1 or Per2 alone do show alterations in anxiety-like behaviors, but the loss of both Per1 and Per2 results in increased anxiety-like behavior. ${ }^{127}$ In human imaging studies of healthy adolescents, PER2 variants have been associated with reward processing in the prefrontal cortex. ${ }^{129}$ Common variants in PER3 have been associated with $\mathrm{BD}^{121,130}$ and multiple related phenotypes including age of onset, post-partum onset, brain white matter integrity, and the rhythms in BD patient fibroblasts. ${ }^{131-135}$ Functional PER3 variants have also been linked with seasonal affective disorders (SADs). ${ }^{131}$

\section{CRY1/2 and FBXL3}

CRY proteins in the nucleus accumbens are involved in depressionlike helpless behaviors, and genetic knockdown of CRY1 in D1 dopamine receptor-containing neurons reduces susceptibility to stress-induced helplessness in mice. ${ }^{136}$ Whole body Cry1-/- mice have increased depression-like behaviors, and Cry1-/-; Cry2-/double knock-out mice have increased anxiety-like behavior. ${ }^{137-139}$ CRY protein is regulated by FBXL3. The Fbxl3 null mutant has increased stabilization of CRY, longer period and reduced anxiety- and depression-like behaviors. ${ }^{140-142}$

\section{NR1D1 and RORA/B}

Stability of REV-ERB $\alpha$ was shown to be lithium-sensitive and SNPs in NR1D1, the gene encoding REV-ERB $\alpha$, had been implicated in lithium responsiveness. ${ }^{143-145}$ Accordingly, there has been considerable interest in the role of REV-ERB $\alpha$ in mood regulation. Rev-erb $\alpha$ null mutant mice have a shorter free-running period, are more sensitive to photic phase advances, and exhibit mania-like behaviors with reduced immobility in the forced swim and tail suspension tests, increased exploratory behavior, hyperactivity, aggression, and motivation. ${ }^{51,146,147}$ A BD-associated variant in RORA was linked with inflexible chronotype. ${ }^{148}$ RORB was associated with pediatric BD in a candidate gene study of 153 family trios. $^{149}$

$D B P$

DBP has been associated with BD in human genetic studies. ${ }^{150-152}$ Dbp null mutant mice are hypoactive and exhibit a shorter wheelrunning period in constant conditions, and become hyperactive after exposure to stress or sleep deprivation. ${ }^{151,153}$ 


\section{CSNK1 and GSK3}

PER proteins are phosphorylated by casein kinases $1 \delta$ and $\varepsilon$ (encoded from CSNK1D/E). Csnk1e overexpressing mice ${ }^{154}$ and Csnk1d knockout mutants ${ }^{155}$ both show mania-like phenotypes. In human subjects, evening chronotype in 107 patients with BD was associated with two intronic variants in CSNK1E. ${ }^{156}$ Another family of protein kinases, GSK3 phosphorylates PER2 and other components of the molecular clock. ${ }^{143,157,158}$ GSK3 overexpressing mice, and mice with mutations rendering GSK3 constitutively active are hyperactive, have a longer circadian period, and exhibit mania-like behaviors. ${ }^{159-161}$ Conversely, pharmacological or genetic inhibition of GSK3 shortens period and phenocopies the mood-stabilizing effects of lithium. ${ }^{162,163}$ Human genetic studies have linked rare duplications of GSK3B to $\mathrm{BD}^{164}$ and common genetic variants in GSK3B to lithium response $\mathrm{e}^{165,166}$ and brain gray matter volume in neuroimaging studies of MDD. ${ }^{167}$

\section{GWAS of clock genes, mood instability, and BD}

GWAS of BD have identified $>60$ gene loci associated with BD. ${ }^{55,168}$ GWAS typically involve very large cohorts and are unbiased with respect to underlying biological pathways, allowing them to identify novel genetic factors. In the most recent GWAS of $>40,000 \mathrm{BD}$ cases and 371,000 controls, genetic risk for BD was associated with decreased morningness and longer sleep duration. Using a novel technique to evaluate genetic dose effects (Mendelian randomization), the association between BD and chronotype was found to be unidirectional, implying a causal role for genetic liability for BD. In contrast, the association between BD and sleep duration was reciprocal implying bidirectional influence. These GWAS of BD did not reveal any single clock gene variant that was genome-wide significant. However, there was enrichment of BD-associated variants in ARNTL. Previous combined GWAS of BD-SCHZ revealed variants in ARNTL distinguished genetic risk for these disorders that is otherwise highly overlapping. ${ }^{168,169}$ A BD-associated risk locus on chromosome 1 is in proximity to CIART and has also been linked with chronotype in multiple GWAS. ${ }^{55,170}$ A disadvantage of GWAS is that in order to assemble large cohorts, there is often loss of phenotypic detail. However, more recent studies have improved upon this shortcoming (e.g., UK Biobank). In addition to being very large, these newer cohorts collect detailed clinical information, and sleep and activity phenotypes, making circadian rhythm analyses of psychiatric disorders possible. ${ }^{171}$

\section{Polygenic risk analyses of $\mathrm{BD}$ and mood instability}

Composite polygenic risk scores (PRS) can be derived from GWAS to estimate the aggregate risk from multiple SNPs. Using accelerometer data from the UK Biobank, Lyall and colleagues examined an objective measure of disrupted circadian rest-activity rhythms, relative amplitude and the relationship to mental health in $>91,000$ individuals from a community sample. ${ }^{172}$ PRS for low relative amplitude was significantly associated with $\mathrm{BD}$, but also with mood instability, neuroticism, and MDD, indicating a non-specific relationship between circadian rhythm disruption and mood. In a related study, GWAS results from $>70,000$ subjects characterized by accelerometry were examined to identify a set of associated genetic markers in NFASC, SLC25A17 and MEIS1 that predict low relative amplitude. ${ }^{173}$ PRS for low relative amplitude was also associated with mood and mental health-related phenotypes including mood instability $(N=78,710)$, diagnosis of $\operatorname{MDD}(N=9543)$, but not with BD $(N=406)$. The relatively small $B D$ sample may have been underpowered to show a specific association with BD diagnosis, but demonstrates the potential for identifying PRS associations between circadian dysfunction and symptom dimensions relevant to BD in very large samples with detailed assessment of sleep and activity.

Using PRS for chronotype, sleep duration, daytime sleepiness, and insomnia derived from GWAS of the UK Biobank cohort, differential genetic loading was examined in BD I and BD II. ${ }^{174}$ The primary cohort included 4672 BD cases and 5714 controls, with a replication cohort of 4366 BD cases and 6091 controls. PRS for sleep duration was associated with BD I and PRS for insomnia was associated with BD II. PRS for morningness was negatively correlated only with BD I in the discovery cohort, but failed replication. These data confirmed that individuals with BD I have significantly greater genetic liability for longer sleep duration, and that BD II shows genetic liability for insomnia. The data regarding genetic liability for chronotype in BD I subjects were suggestive but inconclusive. Notably, sleep and circadian PRS may distinguish BD subtypes.

\section{Summary of vulnerability, heritability and genetic mechanisms of} $B D$

There is evidence that circadian disruption is common in individuals at risk for BD, including behavioral disturbances and increased genetic risk for chronotype and irregular sleep. However, there appears to be no unique association between circadian disruption and BD. Circadian disruption in sleep and behavior appear to be transdiagnostic risk factors affecting not just BD, but also MDD and psychosis. Genetic risk for low amplitude, chronotype and sleep disruption generally indicate considerable overlap across $\mathrm{MDD}, \mathrm{SCHZ}$, and personality traits such as neuroticism and overall well-being. Some findings suggest that circadian rhythms are particularly disrupted in BD of recent onset, possibly increasing the vulnerability to stressful life events that may trigger episode recurrence and/or more severe episodes.

\section{3 | Circadian disruption as a basis for defining sub-groups of BD}

$\mathrm{BD}$ is heterogeneous and exists on a clinical spectrum with subtypes defined by various clinical features, some of which may share common aspects of circadian disruption. ${ }^{175}$ These putative BD subgroups could either include new and/or previously unrecognized groups, or overlap onto existing sub-groups defined by clinical presentation (e.g., age of onset, symptom clusters), clinical course (e.g., predominant polarity, patterns or recurrence/relapse, suicidality), and/or treatment response that differ across dimensions of circadian 
rhythm disruption. There may be particular value in identifying subtypes of BD patients with chronobiological disruption as some may have a more severe illness course, ${ }^{176}$ and/or respond preferentially to chronobiological interventions such as bright light therapy. ${ }^{1}$ To date, only a few studies have explored the feasibility of classifying $\mathrm{BD}$ according to genetic variation in circadian systems, with a particular focus on lithium treatment response and more limited work on clinical features such as mood instability and seasonality.

\subsection{1 | Lithium response}

Lithium responsive BD has been recognized as having distinct clinical features, and genetic underpinnings. ${ }^{177,178}$ The Pharmacogenomics of Bipolar Disorder study (PGBD) was a prospective maintenance trial of lithium monotherapy designed to identify genes associated with clinical response in BD I. ${ }^{178}$ Chronotype at baseline was associated with response, with higher morningness scores predicting favorable outcomes. ${ }^{179}$ In a subset of PGBD subjects, circadian rhythms were studied in cells grown from lithium responders (Li-R) and non-responders (Li-NR). The Per2-luc bioluminescent reporter was used to assess circadian rhythm parameters (amplitude, period and phase) in skin fibroblasts from 59 PGBD participants. ${ }^{179}$ Compared to Li-NR, cells from Li-R had a shorter circadian period and/or were more likely to demonstrate period shortening upon lithium treatment in vitro. Li-NR fibroblasts had a longer period that did not change upon lithium treatment. Another study of BD using patient fibroblasts reported similar results, indicating that cells from BD donors had more variable rhythms compared to controls. In addition, in samples from BD patients the number of mood stabilizing medications of the donor was correlated with period length in the cells. Cells with a long period were less responsive to pharmacological modulation of the circadian rhythms. ${ }^{180}$ Circadian rhythms were also examined in a neurons using stem cell-derived neural progenitor cells (NPC) to grow neurons from controls, Li-R and Li-NR. ${ }^{181}$ Compared to controls and $\mathrm{Li}-\mathrm{R}$, amplitude was reduced in the Li-NR samples. As in fibroblasts, shorter period was observed in NPCs from Li-R versus Li-NR. In single NPCs and neurons, Li-R and Li-NR both showed phase desynchronization compared to controls. These data suggest that trait-like differences in cellular rhythms negatively influence lithium treatment in BD patients, and that one of the mechanisms by which lithium may exert its therapeutic effects is by shifting period. In human studies, the high-risk offspring of BD parents determined to be Li-R or Li-NR have been evaluated longitudinally over two decades. ${ }^{182}$ Compared to controls, the high-risk youth of BD parents had higher rates of sleep disorders, but there was no difference associated with parental history of lithium-response.

\subsection{2 | Seasonality}

Seasonal changes in photoperiod affect day length and require adaptation by circadian rhythms. BD patients commonly show seasonal changes in mood, and SAD occurs more frequently in BD compared to MDD or community controls. One recent study suggested that compared to MDD and BD I, subjects with BD II were the most likely to show seasonal mood variation and disability. ${ }^{183}$ Previous studies of seasonal BD have found association with clock gene variants. In a genetic association study of 269 BD patients ( $26 \%$ with SAD), the authors studied 349 SNPs and after correction for multiple testing, identified significant associations with five variants in NPAS2. ${ }^{184} \mathrm{~A}$ GWAS of SAD composed of both BD and MDD cases and healthy controls did not identify any genome-wide significant associations in clock genes variants, but reported associations with NPAS2, CSNK1E, and RORA in gene set enrichment analyses. ${ }^{185}$ Another study made use of PRS for BD, MDD, and chronotype obtained from GWAS. In 256 MDD and 189 BD patients, there was a significant correlation between seasonal depression and the MDD-PRS, but not BD-PRS or morningness. ${ }^{186}$ Therefore, there is preliminary evidence linking seasonal BD with clock genes, but the evidence is inconsistent.

\subsection{3 | Other sub-phenotypes}

Other potential sub-phenotypes of BD and a relationship to circadian rhythms have been proposed including mixed features ${ }^{187}$ and rapid cycling. ${ }^{188}$ However, these phenotypes have not been fully studied with contemporary methods in large samples and are considered preliminary. Sub-phenotype studies rely upon detailed clinical characterization and large cohorts. As the GWAS increase in size and improve in clinical phenotyping, the ability to perform subgroup analyses of BD may increase.

\subsection{Environmental risk factors for BD}

Environmental factors' impact on circadian rhythm in BD. Light is the most important and best studied environmental cue for influencing circadian rhythms and mood. Meal timing and exercise are also relevant environmental factors but have not been studied in BD from a chronobiological perspective. Bright light therapy is an important feature of this discussion but has been reviewed in detail previously by the CTF and will not be addressed further. ${ }^{1}$

Experimental manipulations of photoperiod. Animal studies permit investigations of light and photoperiod on circadian rhythms and mood, using controlled manipulation of light/dark cycles that would not be possible in humans, including constant darkness (DD) and constant light (LL).

\subsubsection{Animal models of variation in light}

In nocturnal mice, exposure to LL disrupts circadian rhythms of locomotor activity, body temperature, and sleep-wake and causes depressive-like behaviors and anhedonia in mice. ${ }^{63}$ Exposure to dim light at night has also been shown to induce depressive-like 
behaviors in mice as well as induce inflammatory changes in gene expression, decreases in hippocampal neurotrophic factors and cause phase delays in circadian rhythms. ${ }^{64}$ Using experimental photoperiod manipulation, long-day (19L:5D) and short-day (5L:19D) light exposures can model summer and winter photoperiods. Exposure to short- or long-day photoperiods has been found to induce neurotransmitter switching between dopamine and somatostatin in the paraventricular nucleus, affecting depression-like behaviors in adult rats. ${ }^{189}$ Mice with reduced expression of the DAT show mania-like behaviors. DAT mutants have also shown exaggerated affective behaviors with experimental manipulation of photoperiod: more depression-like behaviors under 19L:5D conditions and more impulsivity-like behaviors under 5L:19D conditions. ${ }^{190}$ Compared to control mice, mutants were unable to regulate DAT expression in response to photoperiod changes, suggesting that plasticity in the dopamine system is responsible for fine-tuning reward behaviors in response to changing light conditions. Because mice are a nocturnal species, the correspondence of these findings from mouse experiments to humans remains to be clarified.

\subsection{2 | Human laboratory studies: Light sensitivity in $\mathrm{BD}$}

Supersensitivity of melatonin suppression by light has been proposed as a biomarker for $\mathrm{BD}$. Increased light sensitivity might reflect differences in the function of the eyes, in particular the melanopsincontaining ipRGCs or ipRGC-recipient regions of the retina. The post-illumination pupil response (PIPR) to light has been used as a marker of melanopsin sensitivity. In healthy individuals, PIPR has been found to positively correlate with manic traits. ${ }^{191}$ In contrast, diminished PIPR to blue light was found in patients suffering from seasonal depression compared to healthy controls ${ }^{192}$ and PIPR has been found to vary in winter versus summer in seasonally depressed patients, but not in healthy subjects. ${ }^{193}$ In a study of patients newly diagnosed with $\operatorname{BD}(n=31)$, unaffected relatives $(n=22)$ and controls $(n=35)$, there were no group differences in PIPR, but remitted BD patients showed reduced responses compared to BD patients with active hypomanic/mixed symptoms, suggesting state-dependent differences in BD. ${ }^{194}$ Taken together, the pattern of PIPR findings suggests a possible correlation of retinal light sensitivity with mood symptoms, particularly those for mania. ${ }^{30}$ In vitro studies of human cells show a correlation between intracellular signaling pathways with the effects of light on melatonin secretion, with elevated activity in cells from BD patients compared to controls. ${ }^{195}$ These findings are also supported by GWAS which suggest enrichment of genetic risk factors for BD in light-sensitive signaling pathways. ${ }^{109}$

\subsubsection{Naturalistic studies of light exposure and BD}

Light exposure varies considerably across seasons and geography and could affect the development of photosensitive neural systems underlying mood. A large study of 5536 patients at 50 sites across 32 countries showed that age of BD onset is earlier in places with greater increase in springtime light exposure, ${ }^{196}$ and that more hours of daylight at the birth location during early life is associated with a later onset of BD. ${ }^{197}$ This effect was most pronounced in individuals with $\mathrm{BD} I$ and a positive family history, suggesting the possible presence of a gene $x$ environment interaction. In the same cohort, light exposure was associated with suicidal behaviors. ${ }^{198}$ BD patients living at higher latitude with greater fluctuations in light across seasons were more likely to have experienced a suicide attempt compared to BD patients living in locations where seasonal light changes are less variable. Others have found that greater amount of light in spring/ summer is associated with earlier age of BD onset, and higher rates of hospitalizations for mania. ${ }^{199}$ In a cohort study of 181 BD patients, ambient light exposure was measured for seven consecutive days while measuring symptoms of depression. ${ }^{200}$ After controlling for bedtime, activity, employment and manic symptoms, individuals in the top tercile of light exposure were significantly less likely to be depressed compared to the bottom tercile. Taken together, ambient light may have important effects on mood including onset of manic symptoms and current depressive symptoms.

\subsection{4 | Visual impairment and retinal alterations in $\mathrm{BD}$}

Investigations of individuals with a total absence of light perception or alterations in visual and non-visual pathways could help resolve the relationship between light and BD. Preliminary evidence indicates that congenital blindness may be protective against $\mathrm{SCHZ},{ }^{201}$ but no data are available for BD. One meta-analysis found patients with $\mathrm{BD}$ to have retinal thinning compared to healthy controls. ${ }^{202}$ One study has described impaired color vision in BD. ${ }^{203}$

\subsection{5 | Summary of light exposure studies}

There is reasonable evidence suggesting that light exposure modulates brain circuits regulating mood in BD, and possibly age of onset and suicidality. The underlying mechanisms in humans have not yet been elucidated. There is no conclusive evidence that light exposure or individual differences in light processing play causal roles in BD.

\subsection{Specificity of circadian disruption in BD and across psychiatric disorders}

BD commonly presents with psychiatric comorbidity and is often conceptualized to occur on an illness spectrum. In this way, BD may overlap with other mood and psychotic disorders. The existence of clinical disorders adjacent to BD at diagnostic transitions (e.g., MDD with mixed features, schizoaffective disorder) further underscores this notion and gains additional support from cross-disorder GWAS 
and neuroimaging studies. ${ }^{204,205}$ In genetic studies, BD is shown to have substantial overlap in the risk from common genetic variation with attention deficit hyperactivity disorder (ADHD), obsessivecompulsive disorder (OCD), MDD, and especially SCHZ. ${ }^{205}$ Large structural MRI meta-analyses show substantial correlation in brain structure in BD across SCHZ, OCD, and MDD. ${ }^{204}$ The following section explores the degree to which circadian disruption is specific to $\mathrm{BD}$ and/or is shared across psychiatric disorders.

\subsection{1 | Circadian disruption in high-risk populations}

Two studies reported polysomnography and sleep ratings for 20 help-seeking individuals who met high-risk criteria for psychosis or BD and healthy controls. ${ }^{206,207}$ Compared to controls, individuals in the high-risk group had less daytime activity, longer sleep duration, and lower amplitude motor activity rhythms with more irregular and fragmented rest-activity patterns. However, these results did not control for differences in mood symptoms and chronotypes were similar across groups. There were no differences that distinguished risk for BD versus psychosis.

\subsubsection{Circadian disruption in clinical studies of prodromal psychiatric disorders}

In a study of 248 youth presenting with prodromal psychiatric symptoms, sleep-wake disturbance, social dysfunction, and lack of energy predicted transition to full threshold mental disorders after 1 year, but the relationship was not specific to mood disorders. ${ }^{208} \mathrm{~A}$ later study reported similar findings with poor sleep quality, longer sleep latency, and greater daytime dysfunction among help-seeking, prodromal youth compared to controls, but no specific association with mood disorders or BD. ${ }^{209}$ In a large, 5-year prospective study of $>2300$ youth, sleep/wake disturbance predicted the transition from subthreshold to a syndromic mental disorder. Sleep/wake disturbance was lowest in individuals with non-specific symptoms (7\%), higher in those with subthreshold syndromes (17\%) and highest in those meeting full first-episode criteria for a disorder (32\%). ${ }^{210}$ Regarding BD, mania-like symptoms were one of several predictors of transition, but circadian sleep/wake disturbance showed no specific association with diagnosis. Further analysis identified predictors of transition to psychosis and BD. ${ }^{211}$ Although rates of circadian disruption were nominally higher in individuals who developed $B D$ compared with psychosis ( $42 \%$ vs. $8 \%$ ), the difference was not significant after adjustment for covariates.

A large, longitudinal community cohort study examined associations of insomnia, hypersomnia, and daytime activity with BD onset among $>1800$ youth. ${ }^{212}$ Symptoms at age $15-19$ years were compared with those after the onset of a mental disorder at age 19-25 years. Delayed sleep phase predicted onset of depression, but not mania/hypomania. Hypersomnia and anergia predicted the onset of $\mathrm{BD}$. However, none of these associations were unique to
BD and most showed similar associations with the onset of depressive or psychotic disorders. Similar conclusions were reached by a meta-analysis of 25 unique datasets (including $>45,000$ individuals) that reported sleep disturbance increased the odds for developing a mood or psychotic disorder in early adulthood, with similarly increased risk for BD, psychotic disorders or MDD. ${ }^{213}$

\subsection{3 | Chronotype and actigraphy as predictors of $\mathrm{BD}$}

Among help-seeking youth with subthreshold, fully diagnosed disorders or mixed symptom patterns, individuals with emerging psychosis exhibited elevated morningness scores, while cases with emerging anxiety and depression had low morningness. ${ }^{214}$ Individuals with emerging BD also reported lower morningness than controls, especially in depressed males. Among >1400 students classified as being at high, medium, or low-risk of mental disorders, the high-risk group exhibited low morningness, but this was not uniquely associated with BD. ${ }^{215}$ In 146 subjects with emerging psychiatric disorders assessed with actigraphy, medication use and manic symptoms were independently associated with lower amplitude and circadian disruption regardless of diagnosis. ${ }^{216}$ The largest difference between individuals with emerging BD or MDD, was that relative amplitude was less robust in $\mathrm{BD}$, and that weaker rhythms were associated with increased symptoms. Therefore, preliminary evidence from the cross-disorder studies of high-risk youth indicates that low rhythm amplitude is commonly observed across disorders. Chronotype may distinguish psychosis from BD.

\subsection{4 | Do circadian biomarker studies distinguish BD from other psychiatric disorders?}

The majority of biomarker studies in BD employ a single disorder, case-control design. However, studies of biomarkers comparing BD against additional clinical populations and controls have also examined genetic and molecular aspects of the circadian clock, primarily investigating $\mathrm{SCHZ}$ and MDD, but also ADHD, autism and alcoholism. Among these studies, instances of distinct circadian profiles are reported between BD and comparisons disorders. Candidate gene association studies have shown distinct sets of clock gene SNP associations between BD and MDD. ${ }^{114}$ Among blood biomarkers, BD patients with evening chronotype were shown to have lower serum levels of IL6 and TNF $\alpha$ compared to MDD and controls who did not show any interactions between cytokines and chronotype in a community sample of 213 participants. ${ }^{217}$ With respect to $\mathrm{SCHZ}$, variants in the clock genes ARNTL and the PER3 have been reported to help distinguish the genetic overlap with BD. ${ }^{130,169}$ However, most of these studies reporting BD-specific differences in circadian biomarkers were relatively small, and/or underpowered to distinguish clock associations versus both controls and a comparison group. 
The majority of evidence from better powered human biomarker studies indicates that biological factors underlying circadian disruption in BD are shared across psychiatric disorders. In GWAS of chronotype, PRS for morningness are inversely correlated with risk for BD, but also MDD and SCHZ, and positively correlated with subjective well-being, ${ }^{174,218-220}$ while PRS for low amplitude activity are associated with both MDD and BD as well as trait neuroticism. ${ }^{172,173}$ Although chronotype in these studies is not a perfect proxy for circadian clock function, ${ }^{2}$ this genetic evidence indicates that circadian disruption is associated with a variety of psychiatric phenotypes. In a study specifically designed to evaluate cross-disorder enrichment of PRS for chronotype, sleep duration and insomnia across BD, MDD, and $\mathrm{SCHZ}$, there was widespread genetic overlap in all three disorders with each trait. ${ }^{221}$ BD PRS overlap was for chronotype $64 \%$, sleep-duration $76 \%$, and insomnia $78 \%$. While some unique sleep/ circadian loci were found to overlap with each disorder, overall PRS overlap in BD, MDD, and SCHZ was similar in magnitude. In cell lines and post-mortem brain, specific micro-RNAs (miRNAs-29a/c and 106b) have been linked to the circadian clock, and have been independently linked to $\mathrm{BD}, \mathrm{MDD}$, and $\mathrm{SCHZ} .{ }^{222}$ Using peripheral cell cultures and iPSC-derived neurons, circadian rhythm abnormalities have been described in $\mathrm{BD}$, including anomalies in clock gene expression, ${ }^{223}$ period, ${ }^{135,180}$ phase,${ }^{181}$ and low amplitude. ${ }^{181,224,225}$ Similar studies have revealed cellular rhythm abnormalities in $\mathrm{SCHZ},{ }^{226}$ alcoholism, ${ }^{227}$ Alzheimer's Disease, ${ }^{228}$ and ADHD. ${ }^{229}$ However, none of these cell-based studies have compared two or more clinical samples directly, and some of the differences in rhythms parameters are distinct. For instance, in fibroblast studies, differences from controls in $\mathrm{BD}$ studies were seen in period, whereas in $\mathrm{SCHZ}$ differences were observed only in amplitude. In alcoholism, there was no difference between cases and controls, but period length was associated with illness severity. Therefore, it is difficult to make meaningful comparisons across diagnoses.

Methodological differences may further limit comparisons across disorders. For instance, gene expression rhythms measured with polymerase chain reaction typically employ 4-6 h sampling intervals over 1-2 days and provide less precise estimates for rhythm parameters compared to those made using bioluminescent reporters that take frequent measurements in live cells over 5-7 days. Postmortem studies of brain tissues have been developed as another means of assessing circadian biomarkers in humans with psychiatric disorders. In these studies, tissues are collected from individuals at different times of death. By doing so, it is proposed that rhythmically expressed genes can be examined across time at different phases, and the rhythms re-constructed for between-group analyses. These studies indicate widespread disruption of gene expression rhythms are present in the dorsolateral prefrontal cortex (DLPFC), anterior cingulate cortex (ACC), hippocampus, amygdala, nucleus accumbens, and cerebellum in $\mathrm{MDD}^{230}$; DLPFC in $\mathrm{SCHZ}^{231}$; and DLPFC in aged subjects, ${ }^{232}$ with adult sex differences in DLPFC and ACC. ${ }^{233}$ In affected cases compared to controls, these studies generally show loss of rhythms in ensembles of functionally related genes. Notably, in $\mathrm{SCHZ}$ there are ensembles of genes that become rhythmic only in cases and not controls. ${ }^{231}$ These data indicate that loss of rhythm may be pervasive across neuropsychiatric disorders, but that inappropriate gain of rhythm may be another source of circadian disruption. However, as with cell-based studies, comparisons across disorders have not been performed in the same study. Similarly, a detailed comparison of genes affected has not been completed, and only a small number of brain regions have been examined. Therefore, there may be disease-specific signatures of rhythm disruption in the brain that remain undescribed. Interestingly, despite the importance of circadian disruption in $\mathrm{BD}$, post-mortem gene expression studies of $\mathrm{BD}$ samples have not yet been published.

\subsubsection{Summary of comparative biomarker studies across disorders}

With respect to clock gene involvement, the overall conclusion is that circadian disruption shows considerable overlap across disorders, especially among BD, MDD, and SCHZ. This lack of clear diagnostic boundaries is similar to other heterogeneous clinical features associated with $\mathrm{BD}, \mathrm{SCHZ}$ and schizoaffective disorders such as psychosis, cognition, and brain morphology that have been proposed to constitute transdiagnostic "biotypes" ${ }^{234}$ From this perspective, it is possible that circadian disruption may also define a transdiagnostic biotype, but this remains conjecture and awaits experimental validation. Phenotypes differ importantly across psychiatric disorders, and the particular kind of circadian disruption and underlying molecular mechanisms may be important, meaning that psychiatric phenotypes may have both shared and unique features involving circadian rhythms. For instance, amplitude may be affected in one disorder and phase in another, with both negatively affecting sleep. Existing biomarker studies have so far failed to systematically compare molecular-genetic circadian profiles across disorders. Therefore, there may still be unrecognized molecular clock disruption that is specific to BD. Another area for study relates to state-dependent effects observed in BD that distinguish mania and depression. ${ }^{26,27}$ It is unknown how alterations in circadian biomarkers correlate with mood-dependent changes in gene expression and if similar states in other psychiatric disorders (e.g., depression in MDD) would show similar circadian profiles in BD. Preliminary work in this area has implicated rhythmically expressed genes in blood as a means of distinguishing MDD from BD. ${ }^{235}$ Newer modalities such as epigenetics and microbiome analyses have been proposed, but remain to be examined.

\subsection{Circadian disruption over the illness course of BD}

\subsubsection{Circadian disruption in first episode BD}

It has been hypothesized that circadian disruption may trigger first manic or depressive episodes. Longitudinal studies of individuals 
at high-risk for BD commonly include first onset BD episodes. However, these studies differ in the threshold set for diagnosing BD. For instance, some classify BD cases that do not meet full diagnostic criteria (i.e., BD not otherwise specified, NOS) as fully syndromic BD, while others classify BD-NOS cases as "at-risk" and consider only BD-I or II as fully syndromic. Moreover, cohorts may include individuals previously diagnosed with another psychiatric disorder. In a 6-year longitudinal study of 433 offspring of $\mathrm{BD}$ parents (OSBD) who participated in actigraphy and clinical assessment every 2 years, $11 \%$ developed a BD spectrum disorder. ${ }^{236}$ When compared with 314 individuals who did not transition to $B D$, the new onset $B D$ group was more likely to have an evening chronotype, inadequate sleep, prolonged sleep onset, and multiple awakenings. Importantly, both BD and control offspring commonly reported comorbid non-mood disorder diagnoses at baseline, confounding the interpretation. Opposite results were found in a longer, 10-year study of 107 adolescent OSBD, in which $12.8 \%$ of offspring without a mood disorder at baseline transitioned to BD. ${ }^{237}$ Subthreshold manic symptoms proved the strongest predictor of transition while hypersomnia, anergia, and circadian reversal were not associated with transition to BD. Using the Social Rhythm Metric measure of stability of timing of daily activities, ${ }^{238}$ prospective studies of reward sensitivity have been performed on adolescents at high $(n=138)$ or moderate-risk $(n=95)$ for BD. ${ }^{239}$ Over 3 years, irregular daily activities predicted first onset of a BD spectrum disorder in $10 \%$ of the cohort, with higher rates of conversion in the high-risk group (14\% vs. $4 \%$ ) leading the authors to conclude that irregular social rhythms predicted $\mathrm{BD}$ onset only in high-risk individuals. In another study of social rhythms, 414 young adults with BD II or sub-threshold BD were followed for an average of 33 months. ${ }^{240}$ In the sub-threshold BD group, irregular social rhythms significantly predicted subsequent mood episodes (usually depression) and conversion to BD. These findings are consistent with work reporting that life events that disrupt social rhythms often precede the onset of manic episodes in BD I and depressive episodes subthreshold BD and BD II participants. $^{241,242}$

\section{Conversion from MDD to $B D$}

Some studies have specifically examined whether circadian disruption may help identify individuals with depression who later experience hypo/mania. In a community sample of 3021 youth followed for 10 years, baseline symptoms of depression were compared in individuals later diagnosed with MDD $(n=659)$ or $\operatorname{BD}(n=35)$. Diurnal mood variation was associated with transition to $B D$, but no group differences were identified in hypersomnia, daytime activity or atypical symptoms. ${ }^{243}$ In contrast, a 2-year longitudinal study of youth identified 50 participants who showed early transition to $B D$, then matched them with 50 individuals with MDD. ${ }^{244}$ In this slightly larger, matched cohort, atypical symptoms of depression, especially hypersomnia and anergia were specifically associated with transition to BD.

\section{Circadian rhythm disorders and illness severity}

Circadian phase delay toward eveningness has been found in patients with BD and is associated with a more severe illness course. ${ }^{245-248}$ Notably, up to one-third of BD patients have comorbid circadian rhythm disorders, most commonly delayed-sleep phase disorder. ${ }^{249}$ BD patients with circadian rhythm disorders have a significantly worse illness course with earlier age of onset, more personal and family history of suicidal behaviors, higher recurrence rates, and more antidepressant-related manic switches. ${ }^{176,249,250}$ However, the direction of causality and specificity of these comorbidities with BD illness course are not clear. When considering actigraphy studies in exploring the course of BD over time, a systematic review of 27 studies identified rest/activity metrics including total activity, timing, and variability that were consistently associated with transition between different phases of BD that may be predictive of longitudinal course. ${ }^{251}$

\section{Summary of illness course studies}

There is a weak signal for objectively measured longer duration of sleep and for lower amplitude activity in individuals at risk of BD. Limited evidence indicates later rise time, evening chronotype, irregular daytime activity, and/or daytime dysfunction may also characterize those who will progress to BD and/or convert from MDD. However, many of these studies are limited by the participation of symptomatic subjects with subthreshold mood syndromes. Small samples, lack of correction for multiple comparisons and inconsistent application of diagnostic criteria confound interpretation. Studies in later stages of BD suggest circadian disruption is a risk factor for relapse, but do not control for depressive or manic symptoms that confound assessment of circadian disruption. Circadian sleep-wake disorders are associated with relapse, but the extent to which this association is specific is unclear and may be similar to other conditions that undermine stability (e.g., anxiety, substance abuse or pain conditions).

\subsubsection{Circadian disruption in mood states and relapse}

\section{Do phase shifts cause mood episodes in BD?}

Phase shifts have been associated with mood episodes in BD. The question of causality and state versus trait issues have been addressed in variety of ways including the use of animal models, controlled and naturalistic human studies of BD patients, and as a biomarker of pharmacological interventions targeting mood symptoms.

\section{Phase shift studies in animal models}

Both diurnal and nocturnal animals exposed to altered light/dark cycles show circadian disruptions and adverse changes in moodrelated behaviors. For example, rats exposed to phase advances, showed increased anhedonia and anxiety-like behavior. ${ }^{252}$ Similarly, 
the light at night model in mice causes depression and anxiety-like behaviors and induces phase delays. ${ }^{64}$

\section{Controlled human phase shift studies}

Both depressive and manic episodes have been studied. Supporting episodic state-associations, seven BD subjects were tracked longitudinally and spontaneous phase advances of activity and sleep were associated with resolution of depressive episodes. ${ }^{253}$ More recent studies have incorporated gene expression biomarkers to study differences across mood episodes. In BD outpatients, 16 were recruited while manic, 22 while depressed and compared to 19 community controls. Circadian phase was estimated using buccal swabs to collect cells and measure PER1 and NR1D1 gene expression and salivary melatonin collected under dim light. ${ }^{40}$ Compared to depressed BD patients and controls, manic BD patients showed a phase advance in melatonin onset and similar trends in gene expression. There were no significant differences in the depressed subjects. The study did not assess euthymic BD patients. Another biomarker study of circadian phase was conducted in hospitalized BD patients. ${ }^{254}$ Circadian rhythms in 31 episodes of BD were evaluated at admission, at 2-week intervals during hospitalization, and at the time of discharge. Saliva and buccal swabs were obtained at regular time intervals for 2 days, and used to analyze cortisol and clock gene expression in epithelial cells. Compared to controls, manic BD patients showed a $\sim 7 \mathrm{~h}$ phase advance, whereas depressed BD patients showed $4-5 \mathrm{~h}$ phase delays. Interestingly, mixed episodes were associated with 6-7 h phase delays, intermediate between depression and mania. In these symptomatic BD patients, phase shifts tracked closely with mood symptoms and normalized prior to discharge. The authors point out that the $\sim \mathrm{h}$ phase advance associated with mania could also be interpreted as an extreme $\sim 17$ h phase delay, indicating mood symptoms may shift on a continuum going from depression to mixed episodes and mania as phase delays increase. The model potentially offers an explanation as to why manic switches in/out of depression can occur suddenly and why mixed features are typically not sustained, as they exist as transition states. By proposing that individuals demonstrate differences in the potential range of phase shifts, this model was also used to explain differences across mood disorders, whereby BD I patients show the greatest phase shifts (up to $17 \mathrm{~h}$ ), BD II are intermediate and unipolar depressed patients have the smallest phase shifts (only 4-5 h), suggesting both trait and state-like aspects of circadian phase shifting in BD. Additional evidence for phase delays in depression comes from studies of MDD. Three cross-sectional studies of MDD and healthy controls compared various phase angle differences between groups. ${ }^{255-257}$ Each of these small studies found phase differences associated with depressive states that reflect the delay of one or more physiological, circadian rhythms. Another cross-sectional study of 18 depressed women examined the association between depressive symptoms and plasma DLMO and found the severity of depressive symptoms correlated with the degree of misalignment. ${ }^{258}$ In an actigraphy study of the Dutch Bipolar Cohort $(n=107 \mathrm{BD}, 74$ unaffected siblings and 80 control), participants were assessed for symptoms and activity over 14 days. ${ }^{259}$ All groups reported low levels of manic symptoms, but BD cases had significantly high levels of depression. The investigators found a longer sleep duration and later sleep offset in $\mathrm{BD}$ cases compared to unaffected siblings, but these differences were no longer significant after adjusting for depressive symptoms suggesting a state-like change. No other actigraphy parameter differentiated BD cases. Stable, trait-associations have also been reported. An early case-control study compared the urinary levels of 3-methoxy-4-hydr oxyphenylglycol, a metabolite of norepinephrine and motor activity in healthy controls and BD subjects transitioning through manic, depressive and euthymic states ${ }^{260}$; another study measured activity in manic and mixed states following subjects into recovery. ${ }^{261}$ These latter two studies both revealed phase advances spanning manic and depressive states and remission, suggesting a persistently stable circadian trait.

\section{Naturalistic human phase shift studies}

Studies have examined the clinical features of travelers admitted to psychiatric hospitals for mood or psychotic episodes. In a review, there was some indication of increased risk for mood relapse following long distance travel related to circadian misalignment, with depressive episodes possibly more likely with westward travel (requiring a phase delay to synchronize with local time) and manic episodes more likely travelling eastward (requiring a phase advance). ${ }^{262}$ However, the evidence is based on only three studies, none of which were conducted entirely with BD samples, randomization, or control participants who did not experience a mood episode. With seasonal time changes between daylight savings and standard time common in many areas, 1-h phase shifts have also been examined in a large registry study spanning 6 years. Among BD patients, there was no increased risk of hospitalization for mania in the 2 weeks following the time change compared to the 2 weeks before. ${ }^{263}$ Shift work induces phase changes in susceptible people, and BD patients working at night may be at increased risk for relapse. Two case studies have examined shift work and BD. ${ }^{264,265}$ These reports provide preliminary support for phase disruptions causing mood disturbances in BD. Given the prevalence of shift work in modern society, its impact upon people with BD is in urgent need of more study.

\section{Summary of phase shift studies}

We provide tentative support for the hypothesis that circadian phase advances are related specifically to manic phases of BD and phase delays are related specifically to depression. It is unclear if depression in BD and MDD differ regarding phase delays. Further work is needed to determine whether phase changes are traits of $\mathrm{BD}$ reflecting stable vulnerabilities, or if they relate to manic and/or depressive states.

\subsection{3 | Rhythm-modifying effects of mood stabilizing drugs}

\section{Animals and cellular models of mood stabilizers}

Mood stabilizing drugs commonly interact with the molecular components of the circadian clock and their therapeutic properties 
may relate in part to these actions. In the Clock $\Delta 19$ mouse model of mania, lithium administration normalized anxiety-like behaviors, and reduced hyperactivity effects that may occur through normalizing dopamine in the VTA and striatum. ${ }^{54,111-113}$ As described previously, the kinase GSK3 post-translationally regulates several clock proteins. ${ }^{143,266-268}$ Lithium inhibits GSK3 at therapeutically relevant concentrations and affects circadian rhythms in cells from BD patients. ${ }^{135,180,181}$ Consistent with the notion that lithium engages clock proteins as part of the therapeutic mechanism, mutant mice lacking CRY1 and PER2 show distinct anti-depressant responses to lithium. ${ }^{138}$ In Clock $\Delta 19$ mice, selective inhibitors of GSK3 had lithium-like effects in reducing locomotor hyperactivity. ${ }^{269}$ Similar effects were observed with inhibitors of casein kinase 1D, another post-translational regulator of PER2. ${ }^{269}$ Lithium and selective GSK-3 inhibitors increases circadian rhythm amplitude. ${ }^{270}$ However, BD fibroblasts and neurons often lack this amplitude-increasing effect of lithium, speaking against rhythm amplification as a therapeutic drug mechanism. ${ }^{135,181}$ Instead, period change may be more critical. Lithium has been shown to generally lengthen period in cells, rodents, non-human primates and human subjects. ${ }^{135,271-273}$ However, this effect varies across individuals, is concentration dependent and not uniformly observed in vitro at therapeutically relevant concentrations. Unlike lithium, selective GSK3 inhibitors shorten, rather than lengthen, period, a difference that may be related to lithium's ability to also inhibit inositol monophosphatase. ${ }^{179,270,274,275}$ Suggesting an important role for period change in determining therapeutic outcomes, cells from lithium responsive BD patients are more likely to show period shortening effects from therapeutic levels of lithium in vitro whereas cells from lithium non-responders are more likely to show period lengthening. ${ }^{179}$ In agreement with human laboratory studies, shorter period could be expressed as a phase advance under environmental light/dark conditions and would be predicted to have anti-depressant activity.

Valproic acid also has effects on circadian rhythms. ${ }^{276}$ The DAT knockdown mouse (DAT-KD) exhibits hyperactivity, longer circadian period under constant conditions, and has been proposed as a model for mania. ${ }^{277}$ In DAT-KD mice, valproic acid shortened period of wheel running behavior in vivo and PER2 expression in SCN slices in vitro, returning period length to control levels. Similar period shortening effects were observed in BD patient fibroblasts that had a longer period prior to treatment. ${ }^{277}$ Therefore, one common mechanism shared by GSK3 inhibitors and valproic acid is the association with shortening of circadian period and beneficial changes in moodrelated behaviors. Similar to lithium, valproic acid also normalizes the manic-like behavior of the Clock $\Delta 19$ mice via inhibition of histone deacetylase 2 in the VTA. ${ }^{278}$ It has yet to be determined if valproic acid impacts activity or molecular rhythms in these mice.

Mood stabilizing drugs in BD patients and healthy individuals. A small number of clinical studies have shown phase advances with antidepressant interventions in BD, MDD or mixed depressed samples. These include sleep phase advance, sleep deprivation, and tricyclic antidepressants. ${ }^{279-281}$ Other "combination chronotherapeutic approaches" employ phase advance among other interventions such as lithium, phototherapy and sleep deprivation to show synergistic outcomes compared to a single intervention. ${ }^{282,283}$ There is also evidence that a common therapeutic mechanism underlies the fast-acting treatments short-term sleep deprivation, bright light therapy and ketamine. Ketamine and sleep deprivation were found to engage overlapping cortical pathways involved in circadian rhythms and mood. ${ }^{284}$ In a clinical trial of 51 individuals with treatment refractory depression $(N=21$ with $B D)$ and 38 controls, actigraphy was monitored over 3 days before and after ketamine administration. ${ }^{281}$ Baseline circadian rhythm parameters predicted ketamine response. Rapid ketamine responders had advanced phase rhythms at baseline and on day 1 after treatment compared to ketamine non-responders or subjects who received placebo infusions. On day 3, the ketamine-responders also showed a higher amplitude activity rhythm and more activity overall compared to non-responders. Melatonin may also play a role in the therapeutic actions of mood stabilizers in BD. Both lithium and valproate reduce melatonin suppression in response to light in healthy volunteers. ${ }^{285,286}$ However, there was no effect of lithium on melatonin sensitivity reported in a sample of BD patients. ${ }^{35}$

Summary: Rhythm-modifying effects of mood stabilizing drugs. Many mood stabilizing drugs have effects on circadian rhythms, and it remains plausible that interventions that advance phase (or shorten period) may have value in bipolar depression and that phase delaying (or period lengthening) effects may have anti-manic effects. However, these findings are largely from animal studies and evidence supporting these mechanisms in humans is largely missing. Importantly, many mood stabilizers have both anti-manic and anti-depressant properties but can only affect rhythms in one direction at a time. Any explanation of circadian rhythm effects of drugs must account for this discrepancy. Overall, circadian rhythm modulation as a necessary component of mood stabilizer treatment for BD is weakly supported. Nonetheless, drug treatments that target the circadian clock in BD have a strong theoretical basis and should be studied further.

\section{7 $\quad$ Summary of results}

Sections 3.1 through 3.6 above highlight a significant body of research characterizing the chronobiological underpinnings of BD. Before critical integration of the evidence base in the Discussion below, we briefly note here key findings in each area.

\section{4 | DISCUSSION}

The preceding findings represent the summaries of the CTF, expertisematched into four method-specific workgroups to conduct inclusive reviews of evidence for circadian associations with BD. Considered together, the findings of this review provide substantial evidence for a plurality of associations between circadian rhythm parameters and 
BD-related variables. Key results and conclusions are summarized above (Table 2). The following discussion provides a critical analysis of this evidence, and based upon these conclusions, aims to map an agenda for future research.

\subsection{Evidence for associations between circadian rhythms and BD}

Conclusions of the CTF align broadly with previous literature reviews, and do not undermine growing interest in circadian assessment and intervention for people with BD.,2 However, the present critical review more explicitly recognizes significant uncertainties in the evidence base (see Table 3). People at risk for BD commonly show sleep problems, low amplitude rhythms, and distinct chronotypes as early indicators. Circadian rhythms link multiple relevant neurobiological pathways with strong evidence for involvement in BD. Furthermore, photoreceptive inputs to the $\mathrm{SCN}$ and other brain structures play an important role in mood regulation, especially in animal models, while detailed mapping of photic input pathways have not been well-studied in humans. Strong evidence supports that genetic liability affecting chronotype, sleep and activity parameters intersect with genetic risk for psychiatric disorders, but not specifically for BD. Evidence is strong that changes in environmental light conditions affect mood. Preliminary and incomplete evidence indicates that light supports euthymia when optimally delivered but might provoke mood episodes when exposure is suboptimal and/or misaligned with circadian phase. The majority of evidence from biomarker and developmental studies of young people indicate that there is no particular enrichment of circadian rhythm disturbances in BD relative to mood or psychotic disorders. Nonetheless, several effective drug treatments for BD including lithium and valproic acid may have therapeutically relevant engagement with the circadian clock. The majority of evidence indicates there are stable trait-like differences in sleep and chronotype in BD but the evidence is often confounded by co-morbid psychiatric conditions and often derived from studies performed outside a controlled laboratory setting. Evidence supporting state-like abnormalities related to mood episodes and phase alignment has been provided, but is comparatively weaker, arising from fewer and smaller studies, with more variable and inconsistent outcomes. Importantly, the pattern of findings suggests that specificity to $\mathrm{BD}$ is unlikely, and consistent with a range of research outside BD, that measurable abnormalities in circadian function are associated with a range of psychiatric and medical disorders, developmental disorders, neurodegenerative disorders, metabolic disorders, and cancers. In conceptualizing these findings into an integrated model of $\mathrm{BD}$, we have considered the level of organization, disease specificity, and relative importance of circadian function (Figure 3).

\subsection{Limitations of the literature}

As a whole, the literature is diffusely organized, characterized by investigation of a large number of distinct questions, and assessed using different methods in samples selected on diverse criteria. Many of the proxies accepted as measures of circadian rhythms including actigraphy, sleep, and social rhythms are confounded by non-circadian factors (e.g., masking from light, homeostatic sleep pressure, effects of mood state) and/or incompletely understood in terms of underlying chronobiology. Overall, evidence for most conclusions was not robust, with even the stronger findings undermined by key weaknesses (see Table 2). Within studies, controls for alternative explanations are often missing. Cohorts are often small, and replication of specific findings is rare.

Overall, the findings can be best summarized as "a mosaic of evidence" or "dappled evidence" for non-specific involvement of the circadian system in BD. ${ }^{287,288}$ Whether this is deemed compelling or not depends on the robustness of individual pieces of evidence and whether a more theoretically integrated account is possible.

\subsubsection{Avoiding false positive inferences}

In the context of meta-scientific concern about failures to replicate, ${ }^{289}$ a major goal of the CTF was to attend to the risk of drawing false-positive associations (type I error) in the BD literature pertaining to circadian rhythms. We have identified four conceptual ambiguities that together increase the risk of type I error: definitions of circadian constructs, definitions of BD constructs and reliance on associative rather than causal study designs, and robustness of the findings.

\subsection{2 | A plethora of "circadian" variables}

Many variables can plausibly be the focus of chronobiological research. The elasticity of the concept may threaten construct validity across several dimensions. First, due to the masking effects of light, direct measurement of circadian parameters is often problematic in humans and the laboratory procedures available to unmask the endogenous component of 24-h circadian rhythms are complex, and rarely used at scale with clinical samples. Moreover, given the need for synchronization across multiple levels of organization throughout the body (Figure 3), circadian rhythms do not manifest as a single parameter that can be readily measured experimentally. ${ }^{290}$ Similarly, circadian clocks are open systems, and the functions of input pathways (e.g., light) provide additional variables relevant to BD (Figure 3). Finally, common terms such as "circadian dysregulation" and "circadian disruption", are imprecise and lack consensus biological meanings. ${ }^{291}$ Circadian disruption has been operationalized in various ways often implying that a particular BD-circadian association relates to dynamics that are state-like, transient and reversible (e.g., phase alignment), but also trait-like, stable and enduring (e.g., chronotype). ${ }^{2}$ This wide range of referents for "circadian abnormality" gives chronobiologists too many degrees of freedom, and a critical assessment of the literature must be alert to false positive inferences within and across studies. 
TABLE 2 Overview of key results

\section{Section}

3.1. Neurobiology of circadian clocks in bipolar disorder

\section{Summary of reviewed findings}

- The molecular genetics and neuroanatomy of the circadian system are strongly conserved across mammals

- Circadian clocks interface with biological pathways implicated in healthy and disordered mood regulation

- The role of the SCN in direct mood regulation is incompletely described

- Rhythmic physiological, behavioral, and cognitive processes are outputs of circadian clocks and regulated pathways implicated in BD: dopamine, melatonin, inflammation, sleep, and metabolism

- Extrapolation from nocturnal rodents to humans often required

\subsection{Circadian disruption as a predisposing risk factor for} bipolar disorder

A Vulnerability for bipolar disorder

A Heritability of circadian phenotypes and bipolar disorder

A Genetic mechanisms in the molecular clock and risk for bipolar disorder

3.3. Circadian disruption as a basis for defining subgroups of bipolar disorder

3.4. Environmental risk factors for bipolar disorder

3.5. Specificity of circadian disruption in $\mathrm{BD}$ and across psychiatric disorders

3.6. Circadian disruption across the illness course of bipolar disorder

A Circadian disruption in first episode BD
- Proxy markers of circadian rhythms have been examined as risk factors for BD vulnerability in subjects enriched in familial high-risk, affective temperament, and hypomanic symptoms

- The circadian variables have been limited to sleep parameters, chronotype and motor activity rhythms

- BD risk phenotypes are generally associated with evening chronotype, poor sleep quality, and lower amplitude 24-h sleep/activity rhythms

- Large genetic studies indicate high heritability of BD, dozens of high-confidence genetic loci, only sparse evidence directly implicating clock genes in $\mathrm{BD}$

- Considerable genetic overlap across BD, SCHZ, MDD, neuroticism and overall well-being

- Family and twin studies suggest that instability of 24-h activity rhythms and long sleep duration are heritable and overlap with genetic risk for BD

- Heritability estimates of circadian rhythms rely upon indirect proxy measures, typically actigraphy-derived motor activity, and sleep measures (objective or subjective)

- Polygenic genetic risk for chronotype, low amplitude activity rhythms and sleep duration identified as a trans-diagnostic risk factors for BD, MDD, and $\mathrm{SCHZ}$

- No unique association between circadian disruption and BD. Genetics underlying circadian disruption appears to be equally important as risk factors for MDD and $\mathrm{SCHZ}$

- Suboptimal study designs: community samples, comparatively few clinical samples, incomplete psychiatric phenotyping, not powered for cross-disorder analyses

- Some BD subgroups may be more affected by circadian rhythm abnormalities

- Lithium responsiveness is an established BD subtype with GWAS markers to identify lithium responders and non-responders

- Low morningness associated with lithium-nonresponse in clinical trials

- Lithium non-response is associated with longer circadian period, and lower amplitude in human cellular models using BD patient samples

- Inconsistent support in genetic association studies that parameters related to seasonal mood variation in $\mathrm{BD}$ involves clock gene variants

- Light exposure modulates brain circuits regulating affective behaviors

- Early environmental light exposure associated with manic and depressive features of BD course, age of onset, and suicidality

- Underlying mechanisms of light on mood in humans have not been fully elucidated

- No conclusive evidence that light exposure or individual differences in light processing play a causal role in the development of BD

- No studies of other diurnal environmental factors

- Circadian disruption is widely reported across disorders, especially in BD, MDD, and SCHZ

- Biomarker studies have so far failed to systematically compare molecular-genetic circadian profiles across disorders

- State-dependent differences in BD are understudied: unknown if similar states in other psychiatric disorders (e.g., depression in MDD) are similar or distinct

- Longer duration of sleep and lower amplitude reported in individuals at risk of BD

- Later wake time, evening chronotype, irregular daytime activity and/or daytime impairment reported in first onset of BD and/conversion from MDD

- Prodromal BD symptoms commonly include circadian disruptions, but these are not essential for conversion to syndromic BD

- Studies are limited by the participation of symptomatic subjects with subthreshold mood syndromes

- Small samples, statistical power and inconsistent application of diagnoses further limit quality of evidence in first episode studies 
TABLE 2 (Continued)

Section

A Circadian disruption in mood states and relapse
Summary of reviewed findings

- Tentative support that circadian phase advances specifically associated with mania

- Tentative support that circadian phase delays are specifically associated with depression

- Unclear if BD and MDD differ regarding phase delays

- Further work needed on state-trait characteristics of phase shifts and mood in BD

- Circadian disruption is a risk factor for relapse, but most studies do not control for depressive or manic symptoms that confound assessment of circadian rhythms

- Reports of circadian sleep-wake disorders associations with relapse, but no demonstration of specificity compared to other co-morbid conditions

A Rhythm-modifying effects of mood stabilizing drugs
- Mood stabilizing drugs have clear effects on circadian rhythms

- Interventions that advance phase show anti-depressant properties may have value in the depressed phase of BD

- In principle, phase delaying effects may have anti-manic effects, but evidence is weak

- Lack of well controlled and adequately powered human studies

- Lack of clear mechanism linking specific anti-manic and anti-depressant properties of a drug to circadian variables

- Only weak support that circadian rhythm modulation is a necessary component of mood stabilizer treatment in BD

Abbreviations: BD, bipolar disorder; GWAS, genome wide association studies; MDD, major depressive disorder; SCHZ, schizophrenia.

\subsection{3 | A plethora of BD-related constructs}

Within the categorical clinical diagnoses, BD has numerous recognized facets and subtypes, many of which appear in circadian rhythm studies. Alternative schemes including dimensional, prodromal, or illness-spectrum phenotypes are also widely studied (Figure 4). However, relationships among these categorical and dimensional variables are typically undefined and comparisons across schemes are often problematic. Therefore, evidence for association between a circadian parameter and symptom of BD (e.g., manic symptoms) may not be directly comparable to associations between the same circadian parameter and a phenotype measured on other dimensions (e.g., developmental course of BD in high-risk youth). The variety of BD constructs has implications for assessing the literature and associations with circadian rhythms. For example, if a categorical diagnosis of $\mathrm{BD}$ is the phenotype of interest, then evidence related to the specificity of circadian association are of the utmost importance. By contrast, if we adopt a transdiagnostic and dimensional view of BD-related phenotypes, then specificity is less relevant. Depending on the assumptions of the research, circadian disruption has been offered as a means to explain BD in contradicting terms, defined either broadly (e.g., BD spectrum ${ }^{292}$ ), or narrowly (e.g., subtypes of mood disorders $\left.{ }^{175,293}\right)$.

\subsection{4 | Distinct meanings of "association"}

The majority of circadian studies in BD-related literature assess association between variables and therefore suffer from the adage "correlation does not imply causality." At least three types of 'association' between circadian function and BD appear in the literature: biomarkers, endophenotypes and mechanisms. Biomarkers are best understood as sensitive, specific and measurable indicators of a genetic, molecular, or chemical abnormality that reveals some feature of the disorder (e.g., severity, subtypes, response to treatment). Biomarkers often have prognostic value but are not necessarily causally related to a phenomenon. Endophenotypes (intermediate phenotypes) are stable, quantifiable, trait markers of disease susceptibility. Endophenotypes are often dimensional and can be identified either fully or in attenuated form in unaffected relatives of people with the disorder indicating elevated genetic risk. Mechanisms consist of interacting elements or components whose activities and interactions are organized so as to be responsible for a phenomenon. ${ }^{294}$ Putative mechanisms for BD are genes, molecules, cells, brain systems or physiological/behavioral functions subserving mood symptoms. Circadian parameters are frequently described as biomarkers of $\mathrm{BD}$, for example in relation to predicting lithium response ${ }^{179}$; endophenotypes of $\mathrm{BD}$, for example as a marker of reward sensitivity ${ }^{129,239}$ and as part of the mechanism for $\mathrm{BD}$, overlapping with genetic risk for the disorder. ${ }^{173,174}$ These concepts are rarely defined in explicit terms in circadian studies of BD. Given the distinct implications underlying each kind of association, there may be considerable uncertainty when interpreting a significant finding, especially in the absence of consensus understanding of the theoretical framework. For reasons that differ across methodological approaches, studies demonstrating causality are less common or otherwise limited. When causality is demonstrated, it is commonly in the context of a simplified experimental system such as an animal or cellculture model that is deficient in one or more key aspects of validity. For these reasons, even well-designed demonstrations of causality may not apply to patients with BD. In human studies, the randomized clinical trial (RCT) is commonly accepted as providing causal evidence. However, in most cases RCTs cannot provide the neurobiological mechanistic detail to prove irrefutably that a circadian mechanism is involved unless particular care is taken to study these features simultaneously. Few human circadian studies of BD employ the RCT design in any form. The commonly used cross-sectional, observations with case-control designs typically cannot determine causality. For these reasons, human studies in BD frequently overstate the certainty to 
TABLE 3 Associations between circadian function and bipolar disorder: Conclusions and methodological limitations across themes

\begin{tabular}{|c|c|c|}
\hline Theme & Key conclusions & Methodological limitations of conclusions \\
\hline $\begin{array}{l}\text { Clinically distinct sub- } \\
\text { groups of BD }\end{array}$ & $\begin{array}{l}\text { - Morningness predicts lithium responsiveness, potentially } \\
\text { underpinned by shorter period cellular rhythms }\end{array}$ & $\begin{array}{l}\text { - Cellular rhythms in lithium responsivity have } \\
\text { only been investigated small samples } \\
\text { - Sub-phenotypes not studied in large genetic } \\
\text { samples }\end{array}$ \\
\hline Specificity to BD & $\begin{array}{l}\text { - Absence of specific circadian abnormalities to BD diagnosis is } \\
\text { generally observed }\end{array}$ & $\begin{array}{l}\text { - Piecemeal studies focusing on divergent } \\
\text { circadian and sleep parameters } \\
\text { - Biomarker studies have not directly } \\
\text { compared disorders }\end{array}$ \\
\hline Illness course & $\begin{array}{l}\text { - Evening chronotype, poor sleep predicts onset of BD spectrum } \\
\text { disorder in prospective studies } \\
\text { - Evidence that mania is associated with circadian phase advance } \\
\text { (including clock gene expression) } \\
\text { - In one study, circadian correlates of BD became non-significant } \\
\text { after adjusting for depressive symptoms } \\
\text { - Animal studies suggest altered light/dark cycles disrupt circadian } \\
\text { - Aunction and mood } \\
\text { - Animal and cellular models suggest lithium operates partly } \\
\text { - Clinical studies suggest effective mood stabilizer interventions } \\
\text { are associated with phase advances }\end{array}$ & $\begin{array}{l}\text { - Inconsistent findings across studies } \\
\text { - Limited investigations of mood state changes } \\
\text { with circadian phase shifts } \\
\text { - Limited consensus in replications } \\
\text { - Causality difficult to investigate in humans, } \\
\text { particularly because of third variables } \\
\text { (e.g., stress impacting mood and circadian } \\
\text { rhythms) } \\
\text { - Naturalistic studies have been idiosyncratic } \\
\text { with poor controls }\end{array}$ \\
\hline
\end{tabular}

Abbreviation: BD, bipolar disorder.

which circadian mechanisms are responsible for explaining a particular finding.

\section{3 | Principles for a future research agenda}

\subsection{1 | Unresolved scientific issues}

The review has identified a number of central questions that remain unanswered. A selection of salient questions spanning various levels of scientific inquiry is outlined below (Table 4). Future experimentation to address these within each domain and across domains is high priority.

\subsection{2 | Methodological gaps in the literature}

Compared to other areas of neuroscience research in BD, chronobiology has relatively little research using MRI, PET and other human brain imaging methods, likely due to logistical hurdles associated with performing time course studies with these methods. Brain imaging has the potential to fill an important gap at the junction of neuroanatomy and behavior in BD patient populations and should be encouraged. Other novel methods are beginning to be used in psychiatric populations such as epigenetics, microbiome analyses, and metabolomics but have not yet made an impact on circadian rhythm research in $\mathrm{BD}$.

\subsection{3 | Conceptual advances}

To advance the field, we propose that future research should be conducted in a more systematic and coordinated manner, premised on attention to empirical gaps, methods and theory. At the methodological level, replication must be a priority. Future empirical studies should adopt the three core open science principles of pre-registration, full disclosure of positive and negative findings, and replication as foundations for incremental progress. The definitional and measurement 

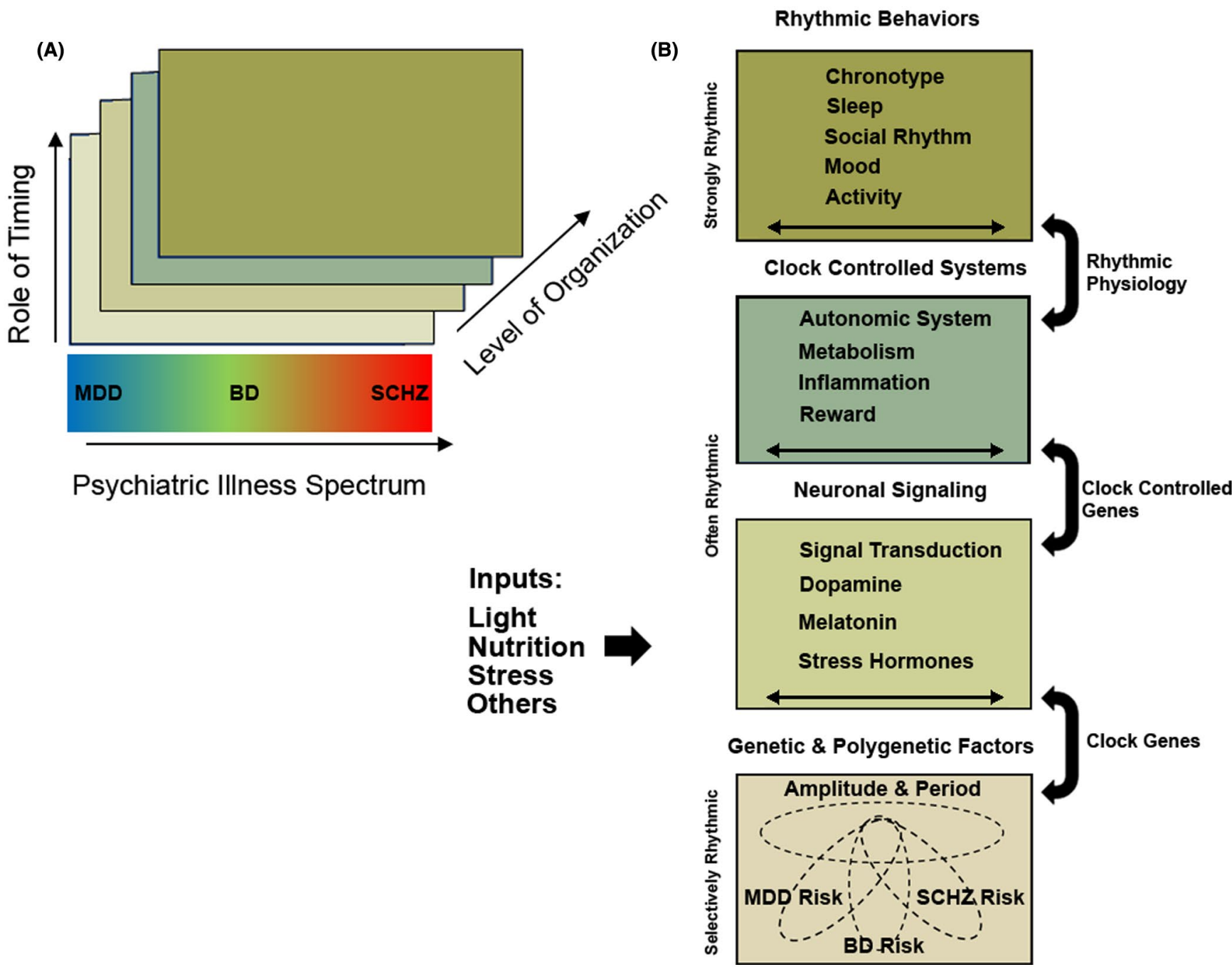

FIGURE 3 Organizational scheme of circadian rhythm disruption in bipolar disorder (BD). (A) Circadian rhythms are manifest across multiple levels of biological and behavioral analysis, starting from genes and progressing upwards into rhythmic output pathways, systems and behaviors. At each level, there exists a spectrum for which disrupted circadian rhythms may cause a psychiatric illness including BD, but also major depressive disorder (MDD), schizophrenia (SCHZ) or features of personality and temperament (not shown). Simultaneously, there exists an independent dimension that defines the importance of circadian regulation for particular system. (B) An expanded description of the scheme is shown with examples at each level. Colors are coded to correspond to levels in panel (A). As the elements of rhythmic behaviors in BD are followed across lower levels of analysis, the set of rhythmic functions becomes more restricted: Circadian rhythms are important for all rhythmic behaviors, most physiological processes under many circumstances, but only a subset of BD and other psychiatric risk genes. At each level, risk for psychiatric disorders exists across the spectrum to varying extents that are not well understood presently. Horizontal arrows indicate that the processes within a level cover a range of conditions within the spectrum of psychiatric disorders, encompassing but not necessarily specific for BD. Psychiatric disorders have discrete genetic factors, but also overlap with each other and with genetic components of the circadian clock. Environmental inputs affect the function of these systems typically at the level of outputs (affecting photoreceptors, nutrient sensors, etc.). Levels are reciprocally interconnected with activity at one level affecting others above and below

issues considered here (Figures 4 and 5) might be useful aids to specifying unambiguous hypotheses. Consensus among researchers on which methods and instruments are best suited for widespread use (e.g., via a formal Delphi study) may improve comparison across studies by harmonizing outcome variables. Structural changes in the systems of research funding, publication, and career advancement may be required to fully implement some of these principles.

At the level of theory, we propose that future studies consider the design within a broad framework. Since different associations between circadian function and BD require different kinds of assessment (Figure 5), we also propose that studies be explicit about the type of association they are investigating (categorical, dimensional, biomarker, endophenotype, mechanism) and when possible, design studies to test causal relationships.

Finally, we note that the literature on circadian associations with BD phenotypes is almost completely silent on the question of integration across the various levels of experimentation. This is remarkable given that, relevant scientific data for the association 
between circadian rhythms and BD ranges from the very small (e.g., gene expression) through mid-level (e.g., brain circuits), to the whole organism (e.g., locomotor activity) and beyond (e.g.,

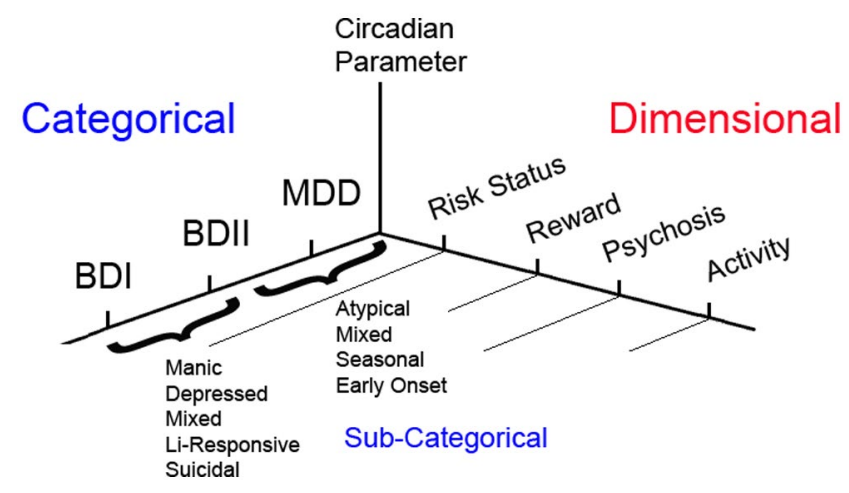

FIGURE 4 Dimensional, categorical, and sub-categorical constructs used in bipolar disorder (BD). Example categorical, sub-categorical and dimensional measures of BD and related mood disorders are shown. The number of categories allows for high degrees of freedom and increases the risk for false positive association (type 1 error). Dimensional constructs can in theory relate to categorical variables, but in practice are not well defined across schemes. The situation makes comparisons across the literature problematic, even when the same parameter of circadian rhythm is employed (e.g., chronotype, social rhythm metric, relative amplitude)

TABLE 4 Questions for future research population studies). Without synthesis across these levels, it is difficult to draw conclusions explaining how the circadian system is involved in BD. Future research should consider ways by which different study designs may be coordinated, adopt scalable measures, and close the gaps in theoretical frameworks. Open data access, resource sharing and promoting research across disciplines may advance these goals.

Explanatory integration is a long-standing aim of the neurobehavioral sciences and there is growing interest in theoretical work to achieve this through 'new mechanistic' and dynamic systems approaches. ${ }^{294,295}$ In this context, it is noteworthy that the circadian system has been presented as an example of the new approach to mechanistic explanation in biology potentially providing the robust theoretical grounding that could inform the integrative synthesis of currently disparate findings. ${ }^{296,297}$ Beyond providing philosophical rigor, efforts toward a cohesive testable multi-level synthesis of circadian regulation of mood would generate new questions, methodological challenges, and clinically relevant insights.

\subsection{Limitations of the review}

The review had a number of limitations. Firstly, as explicated above (Table 3; Section 4.2), the quality of the conclusions here is dependent on the range and quality of the reviewed literature. Secondly, in

\begin{tabular}{|c|c|}
\hline Domain & Question \\
\hline Genetics & $\begin{array}{l}\text { What is the role of pleiotropy for clock genes involved in mood regulation } \\
\text { (i.e., compared to other functions, is the circadian function of a risk } \\
\text { gene essential to its role in BD)? }\end{array}$ \\
\hline Genetics & $\begin{array}{l}\text { How do we reconcile only modest agreement between human genetic } \\
\text { studies of BD with mutant clock gene models in animals? }\end{array}$ \\
\hline Neuroanatomy & $\begin{array}{l}\text { How does rhythm translate into mood regulation and what brain systems } \\
\text { serve this function? }\end{array}$ \\
\hline Neuroanatomy & $\begin{array}{l}\text { Are brain rhythms per se important for mood regulation or is stable mood } \\
\text { mostly dependent on one or more rhythmic output functions (e.g., } \\
\text { dopamine)? }\end{array}$ \\
\hline Neuroanatomy & In which brain structures are rhythms essential for euthymia? \\
\hline Light & $\begin{array}{l}\text { Do the effects of light on mood in humans involve a circadian } \\
\text { mechanism? }\end{array}$ \\
\hline Behavior & $\begin{array}{l}\text { What rhythm parameters best predict diagnosis, mood state, illness } \\
\text { course? }\end{array}$ \\
\hline Behavior & $\begin{array}{l}\text { To what extent do sleep/activity rhythms relate to rhythmic changes in } \\
\text { mood, affect, and reward? }\end{array}$ \\
\hline Behavior & $\begin{array}{l}\text { What are the effects of shift work, jet lag, light at night, timing of social } \\
\text { interactions on first episode, relapse, therapeutic outcomes in } \\
\text { patients with BD? }\end{array}$ \\
\hline Behavior & $\begin{array}{l}\text { How do activity/sleep rhythm parameters (amplitude, phase, variability) } \\
\text { relate to more direct measures of clock function (core body } \\
\text { temperature) in humans? }\end{array}$ \\
\hline Therapeutics & $\begin{array}{l}\text { Do circadian biomarkers or behavioral phenotypes provide clinically } \\
\text { actionable information? }\end{array}$ \\
\hline Therapeutics & Are drugs that target molecular clock mechanisms therapeutic in BD? \\
\hline
\end{tabular}

Abbreviation: BD, bipolar disorder. 

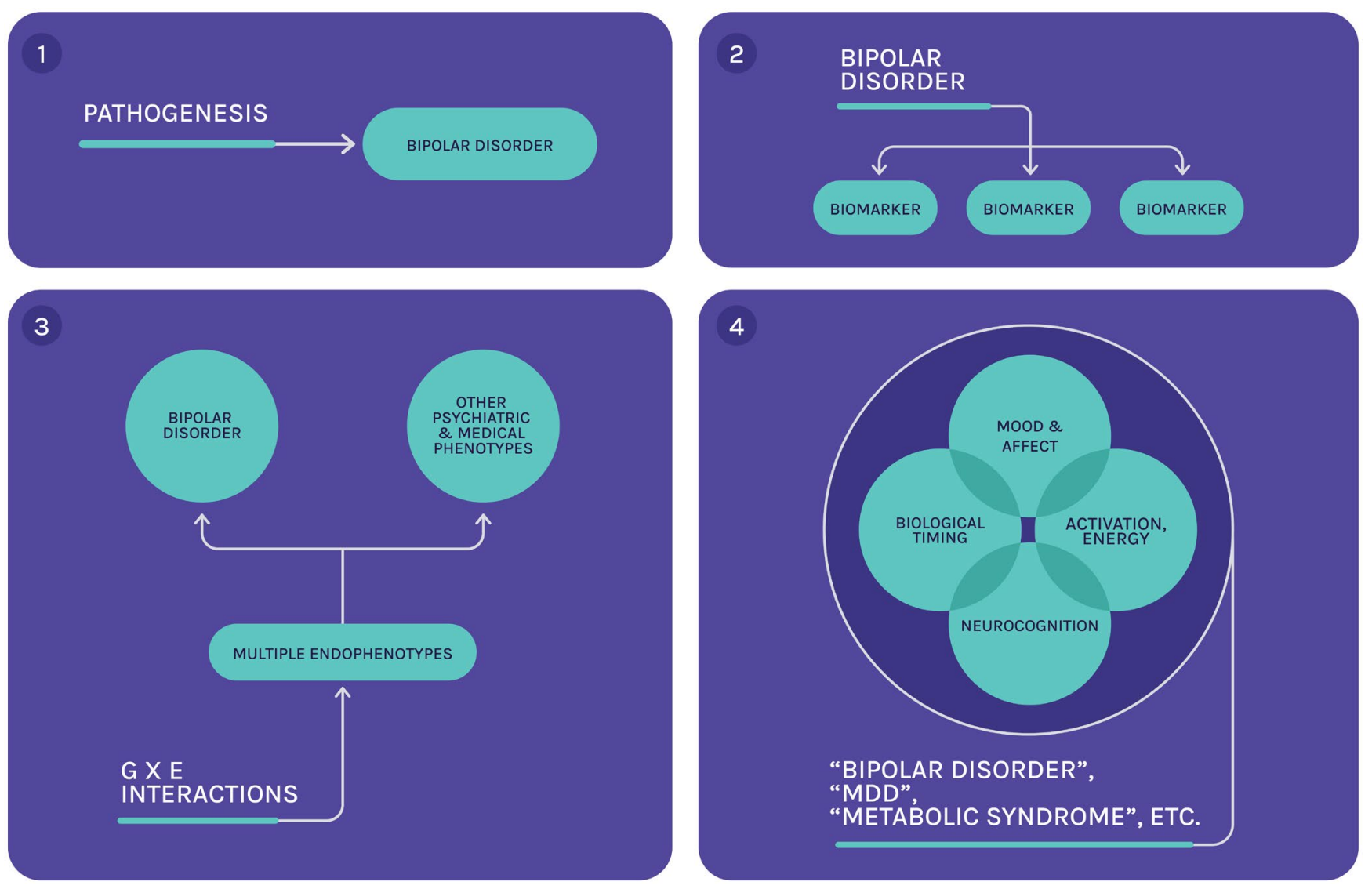

FIGURE 5 Four approaches to conceptualizing circadian involvement in bipolar disorder (BD). (1) Circadian function as causal explanation for emergence of disorder (specificity important, cause important); (2) Circadian function as biomarker (specificity and sensitivity important, cause irrelevant); (3) Circadian function as endophenotype (quantitative and dimensional, specificity not important, causality important, especially across developmental timeframes); (4) Circadian function as a component mechanism ("biological timing") in a complex system, the phenotype of which meets criteria for a variety of diagnoses including BD (specificity not applicable; causality is likely recursive and/or nonlinear)

the absence of a systematic step in the current review, it is possible that key studies were overlooked or under-emphasized, and our conclusions await replication using other literature integration methods. It is hoped that the present critique of terminology and methodology will inform future efforts to use the literature to address questions about the chronobiology of BD. As a whole, the field is often limited by poorly defined variables and the associated effects on theoretical implications. Finally, the evidence reviewed suggests an absence of specificity, and implies that studies using BD diagnosis as their primary criterion relative to other mood disorders may be insensitive to identifying some circadian rhythm associations.

\section{5 | Conclusions}

Within its limitations, the present critical review identifies a range of divergent data, broadly consistent with circadian associations with numerous and variably defined BD phenotypes. The balance of evidence suggests (consistent with the trend away from binary diagnoses) that this association is not specific to BD and extends at least to other psychiatric vulnerabilities. Existing literature is, however, limited in range and methodological quality, and to date there has been little effort towards a rigorous theoretical synthesis. In sum, the present review provides optimism that a future research program addressing these limitations is warranted.

\section{ACKNOWLEDGMENTS}

MJM is supported by a VA Merit Award BX003431. AP is supported by NCCIH (K99 AT010903). DD is supported by the Kavli Institute for Brain and Mind. DL is supported by the German Research Foundation, Emmy Noether Fellowship: LA4126/1-1. LBA is supported by NIMH R01 grants MH077908, MH102310, and MH126911.

\section{CONFLICT OF INTEREST}

H.A.S. receives royalties from Wolters Kluwer and American Psychiatric Association Publishing, an editorial stipend from American Psychiatric Association Publishing, and consulting fees from Novus Medical Education and Medscape. R.J.P. uses computer software provided by SBT-pro at no cost and has received support for travel to educational meetings from Servier and Lundbeck. 


\section{DATA AVAILABILITY STATEMENT}

Data sharing not applicable-no new data generated.

\section{ORCID}

John F. Gottlieb (D) https://orcid.org/0000-0002-2838-8567

Colleen A. McClung (D) https://orcid.org/0000-0003-4719-6197

Bruno Etain (D) https://orcid.org/0000-0002-5377-1488

Dominic Landgraf (D) https://orcid.org/0000-0002-1328-1871

Richard Porter (D) https://orcid.org/0000-0002-8695-3966

Jan Scott (D) https://orcid.org/0000-0002-7203-8601

Holly A. Swartz (D) https://orcid.org/0000-0002-7611-9900

Greg Murray (D) https://orcid.org/0000-0001-7208-5603

\section{REFERENCES}

1. Gottlieb JF, Benedetti F, Geoffroy PA, et al. The chronotherapeutic treatment of bipolar disorders: a systematic review and practice recommendations from the ISBD task force on chronotherapy and chronobiology. Bipolar Disord. 2019;21(8):741-773.

2. Murray G, Gottlieb J, Hidalgo MP, et al. Measuring circadian function in bipolar disorders: empirical and conceptual review of physiological, actigraphic, and self-report approaches. Bipolar Disord. 2020;22(7):693-710.

3. Gold AK, Kinrys G. Treating circadian rhythm disruption in bipolar disorder. Curr Psychiatry Rep. 2019;21(3):14.

4. Malhi GS, Bell E, Bassett D, et al. The 2020 Royal Australian and New Zealand College of Psychiatrists clinical practice guidelines for mood disorders. Aust N Z J Psychiatry. 2021;55(1):7-117.

5. Moher D, Liberati A, Tetzlaff J, Altman DG. Preferred reporting items for systematic reviews and meta-analyses: the PRISMA statement. PLoS Medicine. 2009;6(7):e1000097.

6. Mure LS, Le HD, Benegiamo G, et al. Diurnal transcriptome atlas of a primate across major neural and peripheral tissues. Science. 2018;359(6381):eaao0318.

7. Parekh PK, Logan RW, Ketchesin KD, et al. Cell-type-specific regulation of nucleus accumbens synaptic plasticity and cocaine reward sensitivity by the circadian protein, NPAS2. J Neurosci. 2019;39(24):4657-4667.

8. Garcia JA, Zhang D, Estill SJ, et al. Impaired cued and contextual memory in NPAS2-deficient mice. Science. 2000;288(5474):2226-2230.

9. Albrecht U, Zheng B, Larkin D, Sun ZS, Lee CC. MPer1 and mper2 are essential for normal resetting of the circadian clock. J Biol Rhythms. 2001;16(2):100-104.

10. Landgraf D, McCarthy MJ, Welsh DK. Circadian clock and stress interactions in the molecular biology of psychiatric disorders. Curr Psychiatry Rep. 2014;16(10):483.

11. Hatanaka F, Takumi T. CHRONO integrates behavioral stress and epigenetic control of metabolism. Ann Med. 2017;49(4): 352-356.

12. Goriki A, Hatanaka F, Myung J, et al. A novel protein, CHRONO, functions as a core component of the mammalian circadian clock. PLoS Biol. 2014;12(4):e1001839.

13. Arushanyan EB, Popov AV. Influence of damage to the suprachiasmatic nuclei of the hypothalamus of rats on the dynamics of shortperiod fluctuations of normal and abnormal behavior. Neurosci Behav Physiol. 1995;25(4):290-295.

14. Tataroglu O, Aksoy A, Yilmaz A, Canbeyli R. Effect of lesioning the suprachiasmatic nuclei on behavioral despair in rats. Brain Res. 2004;1001(1-2):118-124.

15. Landgraf D, Long JE, Proulx CD, Barandas R, Malinow R, Welsh DK. Genetic disruption of circadian rhythms in the suprachiasmatic nucleus causes helplessness, behavioral despair, and anxiety-like behavior in mice. Biol Psychiatry. 2016;80(11):827-835.

16. Hattar S, Kumar M, Park A, et al. Central projections of melanopsinexpressing retinal ganglion cells in the mouse. J Comp Neurol. 2006;497(3):326-349.

17. LeGates TA, Fernandez DC, Hattar S. Light as a central modulator of circadian rhythms, sleep and affect. Nat Rev Neurosci. 2014;15(7):443-454.

18. LeGates TA, Altimus CM, Wang $\mathrm{H}$, et al. Aberrant light directly impairs mood and learning through melanopsin-expressing neurons. Nature. 2012;491(7425):594-598.

19. Fernandez DC, Fogerson PM, Lazzerini Ospri L, et al. Light affects mood and learning through distinct retina-brain pathways. Cell. 2018;175(1):71-84 e18.

20. Porcu A, Riddle M, Dulcis D, Welsh DK. Photoperiodinduced neuroplasticity in the circadian system. Neural Plast. 2018;2018:5147585.

21. Kaiser C, Kaufmann C, Leutritz T, Arnold YL, Speck O, Ullsperger $M$. The human habenula is responsive to changes in luminance and circadian rhythm. Neurolmage. 2019;189:581-588.

22. McGlashan EM, Poudel GR, Jamadar SD, Phillips AJK, Cain SW. Afraid of the dark: light acutely suppresses activity in the human amygdala. PLoS One. 2021;16(6):e0252350.

23. Zhang R, Lahens NF, Ballance HI, Hughes ME, Hogenesch JB. A circadian gene expression atlas in mammals: implications for biology and medicine. Proc Natl Acad Sci USA. 2014;111(45):16219-16224.

24. Barandas R, Landgraf D, McCarthy MJ, Welsh DK. Circadian clocks as modulators of metabolic comorbidity in psychiatric disorders. Curr Psychiatry Rep. 2015;17(12):98.

25. Freyberg Z, McCarthy MJ. Dopamine D2 receptors and the circadian clock reciprocally mediate antipsychotic drug-induced metabolic disturbances. NPJ Schizophr. 2017;3:17.

26. McCarthy MJ, Welsh DK. Cellular circadian clocks in mood disorders. J Biol Rhythms. 2012;27(5):339-352.

27. Ketchesin KD, Becker-Krail D, McClung CA. Mood-related central and peripheral clocks. Eur J Neuorsci. 2018;51(1):326-345.

28. McClung CA. How might circadian rhythms control mood? Let me count the ways. Biol Psychiat. 2013;74(4):242-249.

29. Hallam KT, Olver JS, Chambers V, Begg DP, McGrath C, Norman TR. The heritability of melatonin secretion and sensitivity to bright nocturnal light in twins. Psychoneuroendocrinology. 2006;31(7):867-875.

30. Kennedy SH, Kutcher SP, Ralevski E, Brown GM. Nocturnal melatonin and 24-hour 6-sulphatoxymelatonin levels in various phases of bipolar affective disorder. Psychiatry Res. 1996;63(2-3):219-222.

31. Lewy AJ, Nurnberger Jr JI, Wehr TA, et al. Supersensitivity to light: possible trait marker for manic-depressive illness. Am J Psychiatry. 1985;142(6):725-727.

32. Hallam KT, Begg DP, Olver JS, Norman TR. Abnormal doseresponse melatonin suppression by light in bipolar type I patients compared with healthy adult subjects. Acta Neuropsychiatr. 2009;21(5):246-255.

33. Etain B, Dumaine A, Bellivier F, et al. Genetic and functional abnormalities of the melatonin biosynthesis pathway in patients with bipolar disorder. Hum Mol Genet. 2012;21(18):4030-4037.

34. Geoffroy PA, Boudebesse C, Henrion A, et al. An ASMT variant associated with bipolar disorder influences sleep and circadian rhythms: a pilot study. Genes Brain Behav. 2014;13(3):299-304.

35. Nurnberger Jr JI, Adkins S, Lahiri DK, et al. Melatonin suppression by light in euthymic bipolar and unipolar patients. Arch Gen Psychiatry. 2000;57(6):572-579.

36. Ritter P, Wieland F, Skene DJ, et al. Melatonin suppression by melanopsin-weighted light in patients with bipolar I disorder compared to healthy controls. J Psychiatry Neurosci. 2019;44(6):190005. 
37. Whalley LJ, Perini T, Shering A, Bennie J. Melatonin response to bright light in recovered, drug-free, bipolar patients. Psychiatry Res. 1991;38(1):13-19.

38. Lam RW, Berkowitz AL, Berga SL, Clark CM, Kripke DF, Gillin JC. Melatonin suppression in bipolar and unipolar mood disorders. Psychiatry Res. 1990;33(2):129-134.

39. McGlashan EM, Coleman MY, Vidafar P, Phillips AJK, Cain SW. Decreased sensitivity of the circadian system to light in current, but not remitted depression. J Affect Disord. 2019;256:386-392.

40. Novakova M, Prasko J, Latalova K, Sladek M, Sumova A. The circadian system of patients with bipolar disorder differs in episodes of mania and depression. Bipolar Disord. 2015;17(3):303-314.

41. Robillard R, Naismith SL, Rogers NL, et al. Sleep-wake cycle and melatonin rhythms in adolescents and young adults with mood disorders: comparison of unipolar and bipolar phenotypes. Eur Psychiatry. 2013;28(7):412-416.

42. Bucht G, Adolfsson R, Gottfries CG, Roos BE, Winblad B. Distribution of 5-hydroxytryptamine and 5-hydroxyindoleacetic acid in human brain in relation to age, drug influence, agonal status and circadian variation. I Neural Transm (Vienna). 1981;51(3-4):185-203.

43. Green NH, Jackson CR, Iwamoto H, Tackenberg MC, McMahon DG. Photoperiod programs dorsal raphe serotonergic neurons and affective behaviors. Curr Biol. 2015;25(10):1389-1394.

44. Malek ZS, Pevet P, Raison S. Circadian change in tryptophan hydroxylase protein levels within the rat intergeniculate leaflets and raphe nuclei. Neuroscience. 2004;125(3):749-758.

45. Malek ZS, Sage D, Pevet P, Raison S. Daily rhythm of tryptophan hydroxylase-2 messenger ribonucleic acid within raphe neurons is induced by corticoid daily surge and modulated by enhanced locomotor activity. Endocrinology. 2007;148(11):5165-5172.

46. Ashok AH, Marques TR, Jauhar S, et al. The dopamine hypothesis of bipolar affective disorder: the state of the art and implications for treatment. Mol Psychiatry. 2017;22(5):666-679.

47. Berk M, Dodd S, Kauer-Sant'anna M, et al. Dopamine dysregulation syndrome: implications for a dopamine hypothesis of bipolar disorder. Acta Psychiatr Scand Suppl. 2007;434:41-49.

48. Hampp G, Ripperger JA, Houben T, et al. Regulation of monoamine oxidase $A$ by circadian-clock components implies clock influence on mood. Current Biol. 2008;18(9):678-683.

49. Ferris MJ, Espana RA, Locke JL, et al. Dopamine transporters govern diurnal variation in extracellular dopamine tone. Proc Natl Acad Sci USA. 2014;111(26):E2751-E2759.

50. Ozburn AR, Falcon E, Twaddle A, et al. Direct regulation of diurnal Drd3 expression and cocaine reward by NPAS2. Biol Psychiat. 2015;77(5):425-433.

51. Chung S, Lee EJ, Yun S, et al. Impact of circadian nuclear receptor REV-ERBalpha on midbrain dopamine production and mood regulation. Cell. 2014;157(4):858-868.

52. Logan RW, Parekh PK, Kaplan GN, et al. NAD cellular redox and SIRT1 regulate the diurnal rhythms of tyrosine hydroxylase and conditioned cocaine reward. Mol Psychiatry. 2018;24(11):1668-1684.

53. Roybal K, Theobold D, Graham A, et al. Mania-like behavior induced by disruption of CLOCK. Proc Natl Acad Sci USA. 2007;104(15):6406-6411.

54. Sidor MM, Spencer SM, Dzirasa K, et al. Daytime spikes in dopaminergic activity drive rapid mood-cycling in mice. Mol Psychiatry. 2015;20(11):1406-1419.

55. Stahl EA, Breen G, Forstner AJ, et al. Genome-wide association study identifies 30 loci associated with bipolar disorder. Nat Genet. 2019;51(5):793-803

56. Burkeen JF, Womac AD, Earnest DJ, Zoran MJ. Mitochondria calcium signaling mediates rhythmic extracellular ATP accumulation in suprachiasmatic nucleus astrocytes. I Neurosci. 2011;31(23):8432-8440.
57. Schmutz I, Chavan R, Ripperger JA, et al. A specific role for the REV-ERBalpha-controlled L-type voltage-gated calcium channel CaV1.2 in resetting the circadian clock in the late night. J Biol Rhythms. 2014;29(4):288-298

58. McCarthy MJ, Le Roux MJ, Wei H, Beesley S, Kelsoe JR, Welsh DK. Calcium channel genes associated with bipolar disorder modulate lithium's amplification of circadian rhythms. Neuropharmacology. 2016;101:439-448.

59. Isgren A, Sellgren C, Ekman CJ, et al. Markers of neuroinflammation and neuronal injury in bipolar disorder: relation to prospective clinical outcomes. Brain Behav Immun. 2017;65:195-201.

60. Stertz L, Magalhaes PV, Kapczinski F. Is bipolar disorder an inflammatory condition? The relevance of microglial activation. Curr Opin Psychiatry. 2013;26(1):19-26.

61. Giridharan VV, Sayana P, Pinjari OF, et al. Postmortem evidence of brain inflammatory markers in bipolar disorder: a systematic review. Mol Psychiatry. 2020;25(1):94-113.

62. Castanon-Cervantes O, Wu M, Ehlen JC, et al. Dysregulation of inflammatory responses by chronic circadian disruption. Journal of Immunology. 2010;185(10):5796-5805.

63. Fonken LK, Weil ZM, Nelson RJ. Mice exposed to dim light at night exaggerate inflammatory responses to lipopolysaccharide. Brain Behav Immun. 2013;34:159-163.

64. Walker 2nd WH, Borniger JC, Gaudier-Diaz MM, et al. Acute exposure to low-level light at night is sufficient to induce neurological changes and depressive-like behavior. Mol Psychiatry. 2020;25(5):1080-1093.

65. Spengler ML, Kuropatwinski KK, Comas M, et al. Core circadian protein CLOCK is a positive regulator of NF-kappaB-mediated transcription. Proc Natl Acad Sci USA. 2012;109(37):E2457 -E2465.

66. Tang X, Guo D, Lin C, et al. hCLOCK induction by hypoxia promotes inflammatory responses by activating the NFkappaB pathway. Molecular Medicine Reports. 2017;15(3):1401-1406.

67. Okada K, Yano M, Doki Y, et al. Injection of LPS causes transient suppression of biological clock genes in rats. J Surg Res. 2008;145(1):5-12.

68. Marpegan L, Bekinschtein TA, Costas MA, Golombek DA. Circadian responses to endotoxin treatment in mice. J Neuroimmunol. 2005;160(1-2):102-109.

69. Palomba M, Bentivoglio M. Chronic inflammation affects the photic response of the suprachiasmatic nucleus. J Neuroimmunol. 2008;193(1-2):24-27.

70. Lundkvist GB, Hill RH, Kristensson K. Disruption of circadian rhythms in synaptic activity of the suprachiasmatic nuclei by African trypanosomes and cytokines. Neurobiol Dis. 2002;11(1):20-27.

71. Konradi C, Sillivan SE, Clay HB. Mitochondria, oligodendrocytes and inflammation in bipolar disorder: evidence from transcriptome studies points to intriguing parallels with multiple sclerosis. Neurobiol Dis. 2012;45(1):37-47.

72. Clay HB, Sillivan S, Konradi C. Mitochondrial dysfunction and pathology in bipolar disorder and schizophrenia. Int J Dev Neurosci. 2011;29(3):311-324.

73. Marazziti D, Baroni S, Picchetti M, et al. Mitochondrial alterations and neuropsychiatric disorders. Curr Med Chem. 2011;18(30):4715-4721.

74. de Goede P, Wefers J, Brombacher EC, Schrauwen P, Kalsbeek A. Circadian rhythms in mitochondrial respiration. J Mol Endocrinol. 2018;60(3):R115-R130.

75. Asher G, Gatfield D, Stratmann M, et al. SIRT1 regulates circadian clock gene expression through PER2 deacetylation. Cell. 2008;134(2):317-328.

76. Nakahata $\mathrm{Y}$, Kaluzova M, Grimaldi B, et al. The $\mathrm{NAD}^{+}$-dependent deacetylase SIRT1 modulates CLOCK-mediated chromatin remodeling and circadian control. Cell. 2008;134(2):329-340. 
77. Pekovic-Vaughan V, Gibbs J, Yoshitane H, et al. The circadian clock regulates rhythmic activation of the NRF2/glutathione-mediated antioxidant defense pathway to modulate pulmonary fibrosis. Genes Dev. 2014;28(6):548-560.

78. Ishii T, Warabi E, Mann GE. Circadian control of p75 neurotrophin receptor leads to alternate activation of Nrf2 and c-Rel to reset energy metabolism in astrocytes via brain-derived neurotrophic factor. Free Radic Biol Med. 2018;119:34-44.

79. Borbely AA, Daan S, Wirz-Justice A, Deboer T. The twoprocess model of sleep regulation: a reappraisal. J Sleep Res. 2016;25(2):131-143.

80. Ritter PS, Marx C, Lewtschenko N, et al. The characteristics of sleep in patients with manifest bipolar disorder, subjects at high risk of developing the disease and healthy controls. J Neural Transm (Vienna). 2012;119(10):1173-1184.

81. Jones SH, Tai S, Evershed K, Knowles R, Bentall R. Early detection of bipolar disorder: a pilot familial high-risk study of parents with bipolar disorder and their adolescent children. Bipolar Disord. 2006;8(4):362-372.

82. Sebela A, Novak T, Kemlink D, Goetz M. Sleep characteristics in child and adolescent offspring of parents with bipolar disorder: a case control study. BMC Psychiatry. 2017;17(1):199.

83. Wescott DL, Morash-Conway J, Zwicker A, Cumby J, Uher R, Rusak B. Sleep in offspring of parents with mood disorders. Front Psychiatry. 2019;10:225.

84. Scott J, Naismith S, Grierson A, et al. Sleep-wake cycle phenotypes in young people with familial and non-familial mood disorders. Bipolar Disord. 2016;18(8):642-649.

85. Diler RS, Goldstein TR, Hafeman D, et al. Characteristics of depression among offspring at high and low familial risk of bipolar disorder. Bipolar Disord. 2017;19(5):344-352.

86. Soehner AM, Bertocci MA, Manelis A, et al. Preliminary investigation of the relationships between sleep duration, reward circuitry function, and mood dysregulation in youth offspring of parents with bipolar disorder. J Affect Disord. 2016;205:144-153.

87. Meyer TD, Maier S. Is there evidence for social rhythm instability in people at risk for affective disorders? Psychiatry Res. 2006;141(1):103-114.

88. Ankers D, Jones SH. Objective assessment of circadian activity and sleep patterns in individuals at behavioural risk of hypomania. J Clin Psychol. 2009;65(10):1071-1086.

89. Bae M, Lee K, Baek JH, et al. Lifetime experiences of hypomanic symptoms are associated with delayed and irregular sleepwake cycle and seasonality in non-clinical adult samples. Compr Psychiatry. 2014;55(5):1111-1115.

90. Mokros L, Witusik A, Michalska J, et al. Sleep quality, chronotype, temperament and bipolar features as predictors of depressive symptoms among medical students. Chronobiol Int. 2017;34(6):708-720.

91. Bullock B, Murray G. Reduced amplitude of the 24 hour activity rhythm: a biomarker of vulnerability to bipolar disorder? Clin Psychol Sci. 2014;2(1):86-96.

92. Bullock B, Judd F, Murray G. Social rhythms and vulnerability to bipolar disorder. J Affect Disord. 2011;135(1-3):384-388.

93. Indic $P$, Salvatore $P$, Maggini $C$, et al. Scaling behavior of human locomotor activity amplitude: association with bipolar disorder. PLoS One. 2011;6(5):e20650.

94. Rock P, Goodwin G, Harmer C, Wulff K. Daily rest-activity patterns in the bipolar phenotype: a controlled actigraphy study. Chronobiol Int. 2014;31(2):290-296.

95. Hoaki N, Terao T, Wang Y, Goto S, Tsuchiyama K, Iwata N. Biological aspect of hyperthymic temperament: light, sleep, and serotonin. Psychopharmacology. 2011;213(2-3):633-638.

96. Park $\mathrm{Cl}, \mathrm{An} \mathrm{SK}, \mathrm{Kim} \mathrm{HW}$, et al. Relationships between chronotypes and affective temperaments in healthy young adults. J Affect Disord. 2015:175:256-259.
97. Ottoni GL, Lorenzi TM, Lara DR. Association of temperament with subjective sleep patterns. J Affect Disord. 2011;128(1-2):120-127.

98. Merikanto I, Pesonen AK, Kuula L, et al. Eveningness as a risk for behavioral problems in late adolescence. Chronobiol Int 2017;34(2):225-234.

99. McGuffin P, Rijsdijk F, Andrew M, Sham P, Katz R, Cardno A The heritability of bipolar affective disorder and the genetic relationship to unipolar depression. Arch Gen Psychiatry. 2003;60(5):497-502.

100. Coleman JRI, Gaspar HA, Bryois J, Breen G. The genetics of the mood disorder spectrum: genome-wide association analyses of more than 185,000 cases and 439,000 controls. Biol Psychiatry. 2020;88(2):169-184.

101. Ament SA, Szelinger S, Glusman G, et al. Rare variants in neuronal excitability genes influence risk for bipolar disorder. Proc Natl Acad Sci USA. 2015;112(11):3576-3581.

102. Maaser A, Forstner AJ, Strohmaier J, et al. Exome sequencing in large, multiplex bipolar disorder families from Cuba. PLoS One. 2018;13(10):e0205895.

103. Ng TH, Chung KF, Ho FY, Yeung WF, Yung KP, Lam TH. Sleepwake disturbance in interepisode bipolar disorder and high-risk individuals: a systematic review and meta-analysis. Sleep Med Rev. 2015;20:46-58.

104. Gehrman PR, Ghorai A, Goodman M, et al. Twin-based heritability of actimetry traits. Genes Brain Behav. 2019;18(5):e12569.

105. Pagani L., St. Clair P. A., Teshiba T. M., et al. Genetic contributions to circadian activity rhythm and sleep pattern phenotypes in pedigrees segregating for severe bipolar disorder. Proc Natl Acad Sci USA. 2016;113(6):E754-E761.

106. Van Someren EJ, Swaab DF, Colenda CC, Cohen W, McCall WV, Rosenquist PB. Bright light therapy: improved sensitivity to its effects on rest-activity rhythms in Alzheimer patients by application of nonparametric methods. Chronobiol Int. 1999;16(4):505-518

107. Stanislaus S, Vinberg M, Melbye S, et al. Smartphone-based activity measurements in patients with newly diagnosed bipolar disorder, unaffected relatives and control individuals. Int J Bipolar Disord. 2020;8(1):32.

108. Landgraf D, McCarthy MJ, Welsh DK. The role of the circadian clock in animal models of mood disorders. Behav Neurosci. 2014;128(3):344-359.

109. McCarthy MJ. Missing a beat: assessment of circadian rhythm abnormalities in bipolar disorder in the genomic era. Psychiatr Genet. 2019;29(2):29-36.

110. Vitaterna $\mathrm{MH}$, King DP, Chang AM, et al. Mutagenesis and mapping of a mouse gene, Clock, essential for circadian behavior. Science. 1994;264(5159):719-725

111. Roybal K, Theobold D, Graham A, et al. Mania-like behavior induced by disruption of CLOCK. Proc Natl Acad Sci USA. 2007;104(15):6406-6411.

112. Dzirasa K, Coque L, Sidor MM, et al. Lithium ameliorates nucleus accumbens phase-signaling dysfunction in a genetic mouse model of mania. J Neurosci. 2010;30(48):16314-16323.

113. Mukherjee S, Coque L, Cao J-L, et al. Knockdown of Clock in the ventral tegmental area through RNA interference results in a mixed state of mania and depression-like behavior. Biol Psychiatry. 2010;68(6):503-511

114. Soria V, Martinez-Amoros E, Escaramis G, et al. Differential association of circadian genes with mood disorders: CRY1 and NPAS2 are associated with unipolar major depression and CLOCK and VIP with bipolar disorder. Neuropsychopharmacology. 2010;35(6):1279-1289

115. Benedetti F, Dallaspezia S, Fulgosi MC, et al. Actimetric evidence that CLOCK 3111 T/C SNP influences sleep and activity patterns in patients affected by bipolar depression. Am J Med Genet B Neuropsychiatr Genet. 2007;144B(5):631-635. 
116. Benedetti F, Serretti A, Colombo C, et al. Influence of CLOCK gene polymorphism on circadian mood fluctuation and illness recurrence in bipolar depression. Am J Med Genet B Neuropsychiatr Genet. 2003;123B(1):23-26.

117. Lee KY, Song JY, Kim SH, et al. Association between CLOCK 3111T/C and preferred circadian phase in Korean patients with bipolar disorder. Prog Neuropsychopharmacol Biol Psychiatry. 2010;34(7):1196-1201.

118. Kishi T, Yoshimura R, Fukuo Y, et al. The CLOCK gene and mood disorders: a case-control study and meta-analysis. Chronobiol Int. 2011;28(9):825-833.

119. Kondratov RV, Kondratova AA, Gorbacheva VY, Vykhovanets OV, Antoch MP. Early aging and age-related pathologies in mice deficient in BMAL1, the core componentof the circadian clock. Genes Dev. 2006;20(14):1868-1873.

120. Mansour HA, Wood J, Logue T, et al. Association study of eight circadian genes with bipolar I disorder, schizoaffective disorder and schizophrenia. Genes Brain Behav. 2006;5(2):150-157.

121. Nievergelt CM, Kripke DF, Barrett TB, et al. Suggestive evidence for association of the circadian genes PERIOD3 and ARNTL with bipolar disorder. Am J Med Genet B Neuropsychiatr Genet. 2006;141B(3):234-241.

122. Bengesser SA, Reininghaus EZ, Lackner N, et al. Is the molecular clock ticking differently in bipolar disorder? Methylation analysis of the clock gene ARNTL. World J Biol Psychiatry. 2018;19(sup2):S2 1-S29.

123. Qiu P, Jiang J, Liu Z, et al. BMAL1 knockout macaque monkeys display reduced sleep and psychiatric disorders. Natl Sci Rev. 2019;6(1):87-100.

124. Abarca C, Albrecht U, Spanagel R. Cocaine sensitization and reward are under the influence of circadian genes and rhythm. Proc Natl Acad Sci USA. 2002;99(13):9026-9030.

125. Zghoul T, Abarca C, Sanchis-Segura C, Albrecht U, Schumann $G$, Spanagel R. Ethanol self-administration and reinstatement of ethanol-seeking behavior in Per1(Brdm1) mutant mice. Psychopharmacology. 2007;190(1):13-19.

126. Dong L, Bilbao A, Laucht $M$, et al. Effects of the circadian rhythm gene period 1 (per1) on psychosocial stress-induced alcohol drinking. Am J Psychiatry. 2011;168(10):1090-1098.

127. Spencer S, Falcon E, Kumar J, et al. Circadian genes Period 1 and Period 2 in the nucleus accumbens regulate anxiety-related behavior. Eur J Neurosci. 2013;37(2):242-250.

128. Spanagel R, Pendyala G, Abarca C, et al. The clock gene Per2 influences the glutamatergic system and modulates alcohol consumption. Nat Med. 2005;11(1):35-42.

129. Forbes EE, Dahl RE, Almeida JR, et al. PER2 rs2304672 polymorphism moderates circadian-relevant reward circuitry activity in adolescents. Biol Psychiatry. 2012;71(5):451-457.

130. Karthikeyan R, Marimuthu G, Ramasubramanian C, et al. Association of Per3 length polymorphism with bipolar I disorder and schizophrenia. Neuropsychiatr Dis Treat. 2014;10:2325-2330.

131. Zhang L, Hirano A, Hsu PK, et al. A PERIOD3 variant causes a circadian phenotype and is associated with a seasonal mood trait. Proc Natl Acad Sci USA. 2016;113(11):E1536-E1544.

132. Benedetti F, Dallaspezia S, Colombo C, Pirovano A, Marino E, Smeraldi E. A length polymorphism in the circadian clock gene Per3 influences age at onset of bipolar disorder. Neurosci Lett. 2008;445(2):184-187

133. Bollettini I, Melloni EM, Aggio V, et al. Clock genes associate with white matter integrity in depressed bipolar patients. Chronobiol Int. 2017;34(2):212-224.

134. Dallaspezia S, Lorenzi C, Pirovano A, Colombo C, Smeraldi E, Benedetti F. Circadian clock gene Per3 variants influence the postpartum onset of bipolar disorder. Eur Psychiatry. 2011;26(3):138-140
135. McCarthy MJ, Wei H, Marnoy Z, et al. Genetic and clinical factors predict lithium's effects on PER2 gene expression rhythms in cells from bipolar disorder patients. Transl Psychiatry. 2013;3:e318.

136. Porcu A, Vaughan M, Nilsson A, Arimoto N, Lamia K, Welsh DK. Vulnerability to helpless behavior is regulated by the circadian clock component CRYPTOCHROME in the mouse nucleus accumbens. Proc Natl Acad Sci USA. 2020;117(24):13771-13782.

137. De Bundel D, Gangarossa G, Biever A, Bonnefont X, Valjent E. Cognitive dysfunction, elevated anxiety, and reduced cocaine response in circadian clock-deficient cryptochrome knockout mice. Front Behav Neurosc. 2013;7:152.

138. Schnell A, Sandrelli F, Ranc V, et al. Mice lacking circadian clock components display different mood-related behaviors and do not respond uniformly to chronic lithium treatment. Chronobiol Int. 2015;32(8):1075-1089.

139. Huhne A, Volkmann $P$, Stephan $M$, Rossner $M$, Landgraf D. An in-depth neurobehavioral characterization shows anxietylike traits, impaired habituation behavior, and restlessness in male Cryptochrome-deficient mice. Genes Brain Behav. 2020;19(8):e12661.

140. Virshup DM, Forger DB. After hours keeps clock researchers CRYing Overtime. Cell. 2007;129(5):857-859.

141. Anand SN, Maywood ES, Chesham JE, et al. Distinct and separable roles for endogenous CRY1 and CRY2 within the circadian molecular clockwork of the suprachiasmatic nucleus, as revealed by the Fbxl3(Afh) mutation. J Neurosci. 2013;33(17):7145-7153.

142. Keers R, Pedroso I, Breen G, et al. Reduced anxiety and depressionlike behaviours in the circadian period mutant mouse afterhours. PLoS One. 2012;7(6):e38263.

143. Yin L, Wang J, Klein PS, Lazar MA. Nuclear receptor Rev-erbalpha is a critical lithium-sensitive component of the circadian clock. Science. 2006;311(5763):1002-1005.

144. McCarthy MJ, Nievergelt CM, Shekhtman T, Kripke DF, Welsh DK, Kelsoe JR. Functional genetic variation in the Rev-Erbalpha pathway and lithium response in the treatment of bipolar disorder. Genes Brain Behav. 2011;10(8):852-861.

145. Campos-de-Sousa S, Guindalini C, Tondo L, et al. Nuclear receptor rev-erb-\{alpha\} circadian gene variants and lithium carbonate prophylaxis in bipolar affective disorder. J Biol Rhythms. 2010;25(2):132-137.

146. Preitner N, Damiola F, Lopez-Molina L, et al. The orphan nuclear receptor REV-ERBalpha controls circadian transcription within the positive limb of the mammalian circadian oscillator. Cell. 2002;110(2):251-260.

147. Feillet CA, Bainier C, Mateo M, et al. Rev-erbalpha modulates the hypothalamic orexinergic system to influence pleasurable feeding behaviour in mice. Addict Biol. 2015;22(2):411-422.

148. Etain B, Jamain S, Milhiet V, et al. Association between circadian genes, bipolar disorders and chronotypes. Chronobiol Int 2014;31(7):807-814.

149. McGrath CL, Glatt SJ, Sklar P, et al. Evidence for genetic association of RORB with bipolar disorder. BMC Psychiatry. 2009;9:70

150. Morissette J, Villeneuve A, Bordeleau L, et al. Genome-wide search for linkage of bipolar affective disorders in a very large pedigree derived from a homogeneous population in quebec points to a locus of major effect on chromosome 12q23-q24. Am J Med Genet Part A. 1999;88(5):567-587.

151. Le-Niculescu H, McFarland MJ, Ogden CA, et al. Phenomic, convergent functional genomic, and biomarker studies in a stressreactive genetic animal model of bipolar disorder and co-morbid alcoholism. Am J Med Genet B Neuropsychiatr Genet. 2008;147B(2) :134-166.

152. Yamaguchi S, Mitsui S, Yan L, Yagita K, Miyake S, Okamura H Role of DBP in the circadian oscillatory mechanism. Mol Cell Biol. 2000;20(13):4773-4781. 
153. Lopez-Molina L, Conquet F, Dubois-Dauphin M, Schibler U. The DBP gene is expressed according to a circadian rhythm in the suprachiasmatic nucleus and influences circadian behavior. EMBO J. 1997;16(22):6762-6771

154. Bryant CD, Parker CC, Zhou L, et al. Csnk1e is a genetic regulator of sensitivity to psychostimulants and opioids. Neuropsychopharmacology. 2012;37(4):1026-1035.

155. Zhou M, Rebholz H, Brocia C, et al. Forebrain overexpression of CK1delta leads to down-regulation of dopamine receptors and altered locomotor activity reminiscent of ADHD. Proc Natl Acad Sci USA. 2010;107(9):4401-4406.

156. Kripke DF, Nievergelt CM, Joo E, Shekhtman T, Kelsoe JR. Circadian polymorphisms associated with affective disorders. J Circadian Rhythms. 2009;7:2.

157. litaka C, Miyazaki K, Akaike T, Ishida N. A role for glycogen synthase kinase-3beta in the mammalian circadian clock. J Biol Chem. 2005;280(33):29397-29402.

158. Harada Y, Sakai M, Kurabayashi N, Hirota T, Fukada Y. Ser-557phosphorylated mCRY2 is degraded upon synergistic phosphorylation by glycogen synthase kinase-3 beta. J Biol Chem. 2005;280(36):31714-31721.

159. Polter A, Beurel E, Yang S, et al. Deficiency in the inhibitory serine-phosphorylation of glycogen synthase kinase-3 increases sensitivity to mood disturbances. Neuropsychopharmacology. 2010;35(8):1761-1774

160. Prickaerts J, Moechars D, Cryns K, et al. Transgenic mice overexpressing glycogen synthase kinase 3beta: a putative model of hyperactivity and mania. J Neurosci. 2006;26(35):9022-9029.

161. Paul JR, Johnson RL, Jope RS, Gamble KL. Disruption of circadian rhythmicity and suprachiasmatic action potential frequency in a mouse model with constitutive activation of glycogen synthase kinase 3. Neuroscience. 2012;226:1-9.

162. O'Brien WT, Harper AD, Jove F, et al. Glycogen synthase kinase3 beta haploinsufficiency mimics the behavioral and molecular effects of lithium. J Neurosci. 2004;24(30):6791-6798.

163. Kalinichev M, Dawson LA. Evidence for antimanic efficacy of glycogen synthase kinase-3 (GSK3) inhibitors in a strainspecific model of acute mania. Int J Neuropsychopharmacol. 2011;14(8):1051-1067.

164. Lachman HM, Pedrosa E, Petruolo OA, et al. Increase in GSK3beta gene copy number variation in bipolar disorder. Am J Med Genet $B$ Neuropsychiatr Genet. 2007;144B(3):259-265.

165. Benedetti F, Bernasconi A, Lorenzi C, et al. A single nucleotide polymorphism in glycogen synthase kinase 3 -beta promoter gene influences onset of illness in patients affected by bipolar disorder. Neurosci Lett. 2004;355(1-2):37-40.

166. Adli M, Hollinde DL, Stamm T, et al. Response to lithium augmentation in depression is associated with the glycogen synthase kinase 3-beta-50T/C single nucleotide polymorphism. Biol Psychiatry. 2007;62(11):1295-1302

167. Inkster B, Nichols TE, Saemann PG, et al. Association of GSK3beta polymorphisms with brain structural changes in major depressive disorder. Arch Gen Psychiatry. 2009;66(7):721-728.

168. Mullins N, Forstner AJ, O'Connell KS, et al. Genome-wide association study of more than 40,000 bipolar disorder cases provides new insights into the underlying biology. Nat Genet. 2021;53(6):817-829.

169. Ruderfer DM, Ripke S, McQuillin A, Boocock J, Stahl EA. Bipolar disorder and schizophrenia working group of the psychiatric genomics consortium: genomic dissection of bipolar disorder and schizophrenia, including 28 subphenotypes. Cell. 2018;173(7):17051715 e1716.

170. Kalmbach DA, Schneider LD, Cheung J, et al. Genetic basis of chronotype in humans: insights from three landmark GWAS. Sleep. 2017;40(2):zsw048.
171. von Schantz M, Leocadio-Miguel MA, McCarthy MJ, Papiol S, Landgraf D. Genomic perspectives on the circadian clock hypothesis of psychiatric disorders. Adv Genet. 2021;107:153-191.

172. Lyall LM, Wyse CA, Graham N, et al. Association of disrupted circadian rhythmicity with mood disorders, subjective wellbeing, and cognitive function: a cross-sectional study of 91 105 participants from the UK Biobank. Lancet Psychiatry. 2018;5(6):507-514.

173. Ferguson A, Lyall LM, Ward J, et al. Genome-wide association study of circadian rhythmicity in 71,500 UK biobank participants and polygenic association with mood instability. EBioMedicine. 2018;35:279-287.

174. Lewis KJS, Richards A, Karlsson R, et al. Comparison of genetic liability for sleep traits among individuals with bipolar disorder I or II and control participants. JAMA Psychiatry. 2019;77(3):303-310.

175. Gonzalez R, Gonzalez SD, McCarthy MJ. Using chronobiological phenotypes to address heterogeneity in bipolar disorder. Mol Neuropsychiatry. 2020;5(Suppl 1):72-84.

176. Takaesu Y, Inoue $Y$, Ono K, et al. circadian rhythm sleep-wake disorders predict shorter time to relapse of mood episodes in euthymic patients with bipolar disorder: a prospective 48-week study. J Clin Psychiatry. 2018;79(1):17m11565.

177. Hou L, Heilbronner U, Degenhardt F, et al. Genetic variants associated with response to lithium treatment in bipolar disorder: a genome-wide association study. Lancet. 2016.

178. Oedegaard KJ, Alda M, Anand A, et al. The Pharmacogenomics of Bipolar Disorder study (PGBD): identification of genes for lithium response in a prospective sample. BMC Psychiatry. 2016;16:129.

179. McCarthy M, Wei $\mathrm{H}$, Nievergelt $\mathrm{C}$, et al. Chronotype and cellular circadian rhythms predict the clinical response to lithium maintenance treatment in patients with bipolar disorder. Neuropsychopharmacology. 2018.

180. Sanghani HR, Jagannath A, Humberstone T, et al. Patient fibroblast circadian rhythms predict lithium sensitivity in bipolar disorder. Mol Psychiatry. 2021;26(9):5252-5265.

181. Mishra HK, Ying NM, Luis A, et al. Circadian rhythms in bipolar disorder patient-derived neurons predict lithium response: preliminary studies. Mol Psychiatry. 2021.

182. Duffy A, Goodday S, Keown-Stoneman C, Grof P. The emergent course of bipolar disorder: observations over two decades from the canadian high-risk offspring cohort. Am J Psychiatry. 2019;176(9):720-729.

183. Yeom JW, Cho C-H, Jeon S, et al. Bipolar II disorder has the highest prevalence of seasonal affective disorder in early-onset mood disorders: Results from a prospective observational cohort study. Depress Anxiety. 2021;38(6):661-670.

184. Geoffroy PA, Lajnef M, Bellivier F, et al. Genetic association study of circadian genes with seasonal pattern in bipolar disorders. Sci Rep. 2015;5:10232.

185. Ho KWD, Han S, Nielsen JV, et al. Genome-wide association study of seasonal affective disorder. Transl Psychiatry. 2018;8(1):190.

186. Ferrer $\mathrm{A}$, Costas J, Gratacos $\mathrm{M}$, et al. Clock gene polygenic risk score and seasonality in major depressive disorder and bipolar disorder. Genes Brain Behav. 2020;19(8):e12683.

187. Lee HJ, Son GH, Geum D. Circadian rhythm hypotheses of mixed features, antidepressant treatment resistance, and manic switching in bipolar disorder. Psychiatry Investig. 2013;10(3):225-232.

188. Wehr TA. Bipolar mood cycles and lunar tidal cycles. Mol Psychiatry. 2018;23(4):923-931.

189. Dulcis D, Jamshidi P, Leutgeb S, Spitzer NC. Neurotransmitter switching in the adult brain regulates behavior. Science. 2013;340(6131):449-453.

190. Young JW, Cope ZA, Romoli B, et al. Mice with reduced DAT levels recreate seasonal-induced switching between states in bipolar disorder. Neuropsychopharmacology. 2018;43(8):1721-1731. 
191. Bullock B, McGlashan EM, Burns AC, Lu BS, Cain SW. Traits related to bipolar disorder are associated with an increased postillumination pupil response. Psychiatry Res. 2019;278:35-41.

192. Roecklein K, Wong P, Ernecoff N, et al. The post illumination pupil response is reduced in seasonal affective disorder. Psychiatry Res. 2013;210(1):150-158.

193. Roecklein KA, Franzen PL, Wescott DL, et al. Melanopsin-driven pupil response in summer and winter in unipolar seasonal affective disorder. J Affect Disord. 2021;291:93-101.

194. Madsen HO, Ba-Ali S, Heegaard S, et al. Melanopsin-mediated pupillary responses in bipolar disorder-a cross-sectional pupillometric investigation. Int J Bipolar Disord. 2021;9(1):7.

195. Gaspar L, van de Werken M, Johansson AS, et al. Human cellular differences in CAMP-CREB signaling correlate with lightdependent melatonin suppression and bipolar disorder. Eur $J$ Neurosci. 2014;40(1):2206-2215.

196. Bauer M, Glenn T, Alda M, et al. Solar insolation in springtime influences age of onset of bipolar I disorder. Acta Psychiatr Scand. 2017;136(6):571-582.

197. Bauer M, Glenn T, Alda M, et al. Influence of light exposure during early life on the age of onset of bipolar disorder. J Psychiatr Res. 2015;64:1-8.

198. Bauer M, Glenn T, Alda M, et al. Association between solar insolation and a history of suicide attempts in bipolar I disorder. J Psychiatr Res. 2019;113:1-9.

199. Aguglia A, Borsotti A, Cuniberti F, Serafini G, Amore M, Maina $G$. The influence of sunlight exposure on hospitalization in emergency psychiatry. Chronobiol Int. 2017;34(10):1413-1422.

200. Esaki Y, Kitajima T, Obayashi K, Saeki K, Fujita K, Iwata N. Daytime light exposure in daily life and depressive symptoms in bipolar disorder: a cross-sectional analysis in the APPLE cohort. J Psychiatr Res. 2019;116:151-156.

201. Morgan VA, Clark M, Crewe J, et al. Congenital blindness is protective for schizophrenia and other psychotic illness. A wholepopulation study. Schizophr Res. 2018;202:414-416.

202. Hebert M, Gagne AM, Paradis ME, et al. Retinal response to light in young nonaffected offspring at high genetic risk of neuropsychiatric brain disorders. Biol Psychiatry. 2010;67(3):270-274.

203. Garcia-Martin E, Gavin A, Garcia-Campayo J, et al. Visual function and retinal changes in patients with bipolar disorder. Retina. 2019;39(10):2012-2021.

204. Opel N, Goltermann J, Hermesdorf M, Berger K, Baune BT, Dannlowski U. Cross-disorder analysis of brain structural abnormalities in six major psychiatric disorders: a secondary analysis of mega- and meta-analytical findings from the ENIGMA consortium. Biol Psychiatry. 2020;88(9):678-686.

205. Anttila V, Bulik-Sullivan B, Finucane HK, et al. Analysis of shared heritability in common disorders of the brain. Science. 2018;360(6395):eaap8757.

206. Zanini M, Castro J, Coelho FM, et al. Do sleep abnormalities and misaligned sleep/circadian rhythm patterns represent early clinical characteristics for developing psychosis in high risk populations? Neurosci Biobehav Rev. 2013;37(10 Pt 2):2631-2637.

207. Castro J, Zanini M, Goncalves Bda S, et al. Circadian rest-activity rhythm in individuals at risk for psychosis and bipolar disorder. Schizophr Res. 2015;168(1-2):50-55.

208. Grierson AB, Scott J, Glozier N, et al. Can youth at high risk of illness progression be identified by measures of rumination and sleep-wake disturbance. Early Interv Psychiatry. 2019;13(5):1214-1219.

209. Stowkowy J, Brummitt K, Bonneville D, et al. Sleep disturbances in youth at-risk for serious mental illness. Early Interv Psychiatry. 2020;14(3):373-378.

210. Iorfino F, Scott EM, Carpenter JS, et al. Clinical stage transitions in persons aged 12 to 25 years presenting to early intervention mental health services with anxiety, mood, and psychotic disorders. JAMA Psychiatry. 2019;76(11):1167-1175.

211. Carpenter JS, Iorfino F, Cross S, et al. Cohort profile: the Brain and Mind Centre Optymise cohort: tracking multidimensional outcomes in young people presenting for mental healthcare. BMJ Open. 2020;10(3):e030985.

212. Scott J, Byrne E, Medland S, Hickie I. Short communication: self-reported sleep-wake disturbances preceding onset of full-threshold mood and/or psychotic syndromes in community residing adolescents and young adults. J Affect Disord. 2020;277:592-595.

213. Scott J, Kallestad H, Vedaa O, Sivertsen B, Etain B. Sleep disturbances and first onset of major mental disorders in adolescence and early adulthood: a systematic review and meta-analysis. Sleep Med Rev. 2021;57:101429.

214. Fares S, Hermens DF, Naismith SL, White D, Hickie IB, Robillard R. Clinical correlates of chronotypes in young persons with mental disorders. Chronobiol Int. 2015;32(9):1183-1191.

215. Sheaves B, Porcheret K, Tsanas A, et al. Insomnia, nightmares, and chronotype as markers of risk for severe mental illness: results from a student population. Sleep. 2016;39(1):173-181.

216. Robillard R, Oxley C, Hermens DF, et al. The relative contributions of psychiatric symptoms and psychotropic medications on the sleep-wake profile of young persons with anxiety, depression and bipolar disorders. Psychiatry Res. 2016;243:403-406.

217. Mondin TC, de Azevedo CT, Moreira FP, et al. Circadian preferences, oxidative stress and inflammatory cytokines in bipolar disorder: a community study. J Neuroimmunol. 2016;301:23-29.

218. Lane JM, Vlasac I, Anderson SG, et al. Genome-wide association analysis identifies novel loci for chronotype in 100,420 individuals from the UK Biobank. Nat Commun. 2016;7:10889.

219. Hu Y, Shmygelska A, Tran D, Eriksson N, Tung JY, Hinds DA. GWAS of 89,283 individuals identifies genetic variants associated with self-reporting of being a morning person. Nat Commun. 2016;7:10448.

220. Jones SE, Lane JM, Wood AR, et al. Genome-wide association analyses of chronotype in 697,828 individuals provides insights into circadian rhythms. Nat Commun. 2019;10(1):343.

221. O'Connell KS, Frei O, Bahrami S, et al. Characterizing the genetic overlap between psychiatric disorders and sleep-related phenotypes. Biol Psychiatry. 2021;90(9):621-631.

222. Geaghan M, Cairns MJ. MicroRNA and posttranscriptional dysregulation in psychiatry. Biol Psychiatry. 2015;78(4):231-239.

223. Yang S, Van Dongen HP, Wang K, Berrettini W, Bucan M Assessment of circadian function in fibroblasts of patients with bipolar disorder. Mol Psychiatry. 2009;14(2):143-155.

224. Nudell V, Wei H, Nievergelt C, et al. Entrainment of circadian rhythms to temperature reveals amplitude deficits in fibroblasts from patients with bipolar disorder and possible links to calcium channels. Molecular Neuropsychiatry. 2019;5(2):115-124

225. McCarthy MJ, LeRoux M, Wei H, Beesley S, Kelsoe JR, Welsh DK. Calcium channel genes associated with bipolar disorder modulate lithium's amplification of circadian rhythms. Neuropharmacology. 2016;101:439-448.

226. Johansson AS, Owe-Larsson B, Hetta J, Lundkvist GB. Altered circadian clock gene expression in patients with schizophrenia. Schizophr Res. 2016;174(1-3):17-23.

227. McCarthy MJ, Fernandes M, Kranzler HR, Covault JM, Welsh DK. Circadian clock period inversely correlates with illness severity in cells from patients with alcohol use disorders. Alcohol Clin Exp Res. 2013;37(8):1304-1310.

228. Cronin P, McCarthy MJ, Lim AS, et al. Circadian alterations during early stages of Alzheimer's disease are associated with aberrant cycles of DNA methylation in BMAL1. Alzheimers Dement. 2017;13(6):689-700 
229. Coogan AN, Schenk M, Palm D, et al. Impact of adult attention deficit hyperactivity disorder and medication status on sleep/wake behavior and molecular circadian rhythms. Neuropsychopharmacology. 2019;44(7):1198-1206.

230. Li JZ, Bunney BG, Meng F, et al. Circadian patterns of gene expression in the human brain and disruption in major depressive disorder. Proc Natl Acad Sci USA. 2013;110(24):9950-9955.

231. Seney ML, Cahill K, Enwright 3rd JF, et al. Diurnal rhythms in gene expression in the prefrontal cortex in schizophrenia. Nat Commun. 2019;10(1):3355.

232. Chen CY, Logan RW, Ma T, et al. Effects of aging on circadian patterns of gene expression in the human prefrontal cortex. Proc Natl Acad Sci USA. 2016;113(1):206-211.

233. Logan RW, Xue X, Ketchesin KD, et al. Sex differences in molecular rhythms in the human cortex. Biol Psychiatry. 2022;91(1):152-162.

234. Ivleva El, Clementz BA, Dutcher AM, et al. Brain structure biomarkers in the psychosis biotypes: findings from the bipolarschizophrenia network for intermediate phenotypes. Biol Psychiatry. 2017;82(1):26-39.

235. Le-Niculescu H, Roseberry K, Gill SS, et al. Precision medicine for mood disorders: objective assessment, risk prediction, pharmacogenomics, and repurposed drugs. Mol Psychiatry. 2021;26(7):2776-2804.

236. Levenson JC, Axelson DA, Merranko J, et al. Differences in sleep disturbances among offspring of parents with and without bipolar disorder: association with conversion to bipolar disorder. Bipolar Disord. 2015;17(8):836-848.

237. Mesman E, Nolen WA, Keijsers L, Hillegers MHJ. Baseline dimensional psychopathology and future mood disorder onset: findings from the Dutch Bipolar Offspring Study. Acta Psychiatr Scand. 2017;136(2):201-209.

238. Monk TH, Kupfer DJ, Frank E, Ritenour AM. The Social Rhythm Metric (SRM): measuring daily social rhythms over 12 weeks. Psychiatry Res. 1991;36(2):195-207.

239. Alloy LB, Boland EM, Ng TH, Whitehouse WG, Abramson LY. Low social rhythm regularity predicts first onset of bipolar spectrum disorders among at-risk individuals with reward hypersensitivity. $J$ Abnorm Psychol. 2015;124(4):944-952.

240. Shen GH, Alloy LB, Abramson LY, Sylvia LG. Social rhythm regularity and the onset of affective episodes in bipolar spectrum individuals. Bipolar Disord. 2008;10(4):520-529.

241. Sylvia LG, Alloy LB, Hafner JA, Gauger MC, Verdon K, Abramson LY. Life events and social rhythms in bipolar spectrum disorders: a prospective study. Behav Ther. 2009;40(2):131-141.

242. Malkoff-Schwartz S, Frank E, Anderson B, et al. Stressful life events and social rhythm disruption in the onset of manic and depressive bipolar episodes: a preliminary investigation. Arch Gen Psychiatry. 1998;55(8):702-707.

243. Pfennig A, Ritter PS, Hofler M, et al. Symptom characteristics of depressive episodes prior to the onset of mania or hypomania. Acta Psychiatr Scand. 2016;133(3):196-204.

244. Scott J, Marwaha S, Ratheesh A, et al. Bipolar at-risk criteria: an examination of which clinical features have optimal utility for identifying youth at risk of early transition from depression to bipolar disorders. Schizophr Bull. 2017;43(4):737-744.

245. Mansour HA, Wood J, Chowdari KV, et al. Circadian phase variation in bipolar I disorder. Chronobiol Int. 2005;22(3):571-584.

246. Ahn YM, Chang J, Joo YH, Kim SC, Lee KY, Kim YS. Chronotype distribution in bipolar I disorder and schizophrenia in a Korean sample. Bipolar Disord. 2008;10(2):271-275.

247. Wood J, Birmaher B, Axelson D, et al. Replicable differences in preferred circadian phase between bipolar disorder patients and control individuals. Psychiatry Res. 2009;166(2-3):201-209.

248. Boudebesse C, Lajnef M, Geoffroy PA, et al. Chronotypes of bipolar patients in remission: validation of the French version of the circadian type inventory in the FACE-BD sample. Chronobiol Int. 2013;30(8):1042-1049.

249. Takaesu Y, Inoue Y, Ono K, et al. Circadian rhythm sleep-wake disorders as predictors for bipolar disorder in patients with remitted mood disorders. J Affect Disord. 2017;220:57-61.

250. Takaesu Y, Inoue Y, Murakoshi A, et al. Prevalence of circadian rhythm sleep-wake disorders and associated factors in euthymic patients with bipolar disorder. PLoS One. 2016;11(7):e0159578.

251. Scott J, Colom F, Young A, Bellivier F, Etain B. An evidence map of actigraphy studies exploring longitudinal associations between rest-activity rhythms and course and outcome of bipolar disorders. Int J Bipolar Disord. 2020;8(1):37.

252. Horsey EA, Maletta T, Turner H, Cole C, Lehmann H, Fournier NM. Chronic jet lag simulation decreases hippocampal neurogenesis and enhances depressive behaviors and cognitive deficits in adult male rats. Front Behav Neurosci. 2019;13:272.

253. Wehr TA, Wirz-Justice A, Goodwin FK, Duncan W, Gillin JC. Phase advance of the circadian sleep-wake cycle as an antidepressant. Science. 1979;206(4419):710-713

254. Moon $\mathrm{JH}$, Cho $\mathrm{CH}$, Son $\mathrm{GH}$, et al. Advanced circadian phase in mania and delayed circadian phase in mixed mania and depression returned to normal after treatment of bipolar disorder. EBioMedicine. 2016;11:285-295.

255. Hasler BP, Buysse DJ, Kupfer DJ, Germain A. Phase relationships between core body temperature, melatonin, and sleep are associated with depression severity: further evidence for circadian misalignment in non-seasonal depression. Psychiatry Res. 2010;178(1):205-207.

256. Buckley TM, Schatzberg AF, Buckley TM, Schatzberg AF. A pilot study of the phase angle between cortisol and melatonin in major depression-a potential biomarker? J Psychiatr Res. 2010;44(2):69-74.

257. Teicher MH, Lawrence JM, Barber NI, Finklestein SP, Lieberman HR, Baldessarini RJ. Increased activity and phase delay in circadian motility rhythms in geriatric depression: preliminary observations. Arch Gen Psychiatry. 1988;45(10):913-917.

258. Emens J, Lewy A, Kinzie JM, Arntz D, Rough J. Circadian misalignment in major depressive disorder. Psychiatry Res. 2009;168(3):259-261.

259. Verkooijen S, van Bergen AH, Knapen SE, et al. An actigraphy study investigating sleep in bipolar I patients, unaffected siblings and controls. J Affect Disord. 2017;208:248-254.

260. Wehr TA, Muscettola G, Goodwin FK. Urinary 3-methoxy-4-hydroxyphenylglycol circadian rhythm. Early timing (phase-advance) in manic-depressives compared with normal subjects. Arch Gen Psychiatry. 1980;37(3):257-263.

261. Salvatore P, Ghidini S, Zita G, et al. Circadian activity rhythm abnormalities in ill and recovered bipolar I disorder patients. Bipolar Disord. 2008;10(2):256-265.

262. Inder ML, Crowe MT, Porter R. Effect of transmeridian travel and jetlag on mood disorders: evidence and implications. Aust NZ J Psychiatry. 2016;50(3):220-227.

263. Lahti TA, Haukka J, Lönnqvist J, Partonen T. Daylight saving time transitions and hospital treatments due to accidents or manic episodes. BMC Public Health. 2008;8(1):1-4.

264. Meyrer R, Demling J, Kornhuber J, Nowak M. Effects of night shifts in bipolar disorders and extreme morningness. Bipolar Disord. 2009;11(8):897-899.

265. Frank E, Swartz HA, Kupfer DJ. Interpersonal and social rhythm therapy: managing the chaos of bipolar disorder. Biol Psychiat. 2000;48(6):593-604.

266. Sahar S, Zocchi L, Kinoshita C, Borrelli E, Sassone-Corsi P. Regulation of BMAL1 protein stability and circadian function by GSK3beta-mediated phosphorylation. PLoS One. 2010;5(1):e8561. 
267. litaka C, Miyazaki K, Akaike T, Ishida N. A role for glycogen synthase kinase-3beta in the mammalian circadian clock. J Biol Chem. 2005;280(33):29397-29402.

268. Harada Y, Sakai M, Kurabayashi N, Hirota T, Fukada Y. Ser-557phosphorylated mCRY2 is degraded upon synergistic phosphorylation by glycogen synthase kinase- 3 beta. J Biol Chem. 2005;280(36):31714-31721.

269. Kozikowski AP, Gunosewoyo H, Guo S, et al. Identification of a glycogen synthase kinase-3beta inhibitor that attenuates hyperactivity in CLOCK mutant mice. ChemMedChem. 2011;6(9):1593-1602.

270. Hirota T, Lewis WG, Liu AC, Lee JW, Schultz PG, Kay SA. A chemical biology approach reveals period shortening of the mammalian circadian clock by specific inhibition of GSK-3beta. Proc Natl Acad Sci USA. 2008;105(52):20746-20751.

271. Welsh DK, Moore-Ede MC. Lithium lengthens circadian period in a diurnal primate, Saimiri sciureus. Biol Psychiatry. 1990;28(2): 117-126.

272. Kripke DF, Judd LL, Hubbard B, Janowsky DS, Huey LY. The effect of lithium carbonate on the circadian rhythm of sleep in normal human subjects. Biol Psychiatry. 1979;14(3):545-548.

273. Kripke DF, Wyborney VG. Lithium slows rat circadian activity rhythms. Life Sci. 1980;26(16):1319-1321.

274. Wei H, Landgraf D, Wang G, McCarthy MJ. Inositol polyphosphates contribute to cellular circadian rhythms: implications for understanding lithium's molecular mechanism. Cell Signal. 2018;44: 82-91.

275. Berridge MJ, Downes CP, Hanley MR. Neural and developmental actions of lithium: a unifying hypothesis. Cell. 1989;59(3):411-419.

276. Johansson AS, Brask J, Owe-Larsson B, Hetta J, Lundkvist GB. Valproic acid phase shifts the rhythmic expression of Period2:Luciferase. J Biol Rhythms. 2011;26(6):541-551.

277. Landgraf D, Joiner WJ, McCarthy MJ, et al. The mood stabilizer valproic acid opposes the effects of dopamine on circadian rhythms. Neuropharmacology. 2016;107:262-270.

278. Logan RW, Ozburn AR, Arey RN, et al. Valproate reverses manialike behaviors in mice via preferential targeting of HDAC2. Mol Psychiatry. 2021;26(8):4066-4084.

279. Bunney BG, Bunney WE. Rapid-acting antidepressant strategies: mechanisms of action. Int J Neuropsychopharmacol. 2012;15(05): 695-713.

280. Sack DA, Nurnberger J, Rosenthal NE, et al. Potentiation of antidepressant medications by phase advance of the sleep-wake cycle. Am J Psychiatry. 1985;142(5):606-608.

281. Duncan Jr WC, Slonena E, Hejazi NS, et al. Motor-activity markers of circadian timekeeping are related to ketamine's rapid antidepressant properties. Biol Psychiatry. 2017;82(5):361-369.

282. Wu JC, Kelsoe JR, Schachat C, et al. Rapid and sustained antidepressant response with sleep deprivation and chronotherapy in bipolar disorder. Biol Psychiat. 2009;66(3):298-301.

283. Benedetti F, Dallaspezia S, Fulgosi MC, Barbini B, Colombo C Smeraldi E. Phase advance is an actimetric correlate of antidepressant response to sleep deprivation and light therapy in bipolar depression. Chronobiol Int. 2007;24(5):921-937.
284. Orozco-Solis R, Montellier E, Aguilar-Arnal L, et al. A circadian genomic signature common to ketamine and sleep deprivation in the anterior cingulate cortex. Biol Psychiatry. 2017;82(5):351-360.

285. Hallam KT, Olver JS, Horgan JE, McGrath C, Norman TR. Low doses of lithium carbonate reduce melatonin light sensitivity in healthy volunteers. Int J Neuropsychopharmacol. 2005;8(2):255-259.

286. Hallam KT, Olver JS, Norman TR. Effect of sodium valproate on nocturnal melatonin sensitivity to light in healthy volunteers. Neuropsychopharmacology. 2005;30(7):1400-1404.

287. Dubreuil B. Explaining the brain: mechanisms and the mosaic unity of neurosciences, by Carl F. Craver. Eur J Philos. 2010;18(3):471-474

288. Kendler KS. The dappled nature of causes of psychiatric illness: replacing the organic-functional/hardware-software dichotomy with empirically based pluralism. Mol Psychiatry. 2012;17(4):377-388.

289. Shrout PE, Rodgers JL. Psychology, science, and knowledge construction: broadening perspectives from the replication crisis. Annu Rev Psychol. 2018;69:487-510.

290. Roenneberg T, Pilz LK, Zerbini G, Winnebeck EC. Chronotype and social jetlag: a (self-) critical review. Biology (Basel). 2019;8(3):54.

291. Vetter C. Circadian disruption: what do we actually mean? Eur J Neurosci. 2020;51(1):531-550.

292. McCarthy MJ, Nievergelt CM, Kelsoe JR, Welsh DK. A survey of genomic studies supports association of circadian clock genes with bipolar disorder spectrum illnesses and lithium response. PLoS One. 2012;7(2):e32091.

293. Carpenter JS, Crouse JJ, Scott EM, et al. Circadian depression: a mood disorder phenotype. Neurosci Biobehav Rev. 2021;126: 79-101.

294. Glennan S, Illari P. The Routledge Handbook of Mechanisms and Mechanical Philosophy. London, UK: Routledge; 2017.

295. Miłkowski M, Hohol M, Nowakowski P. Mechanisms in psychology: the road towards unity? Theory Psychol. 2019;29(5):567-578.

296. Bechtel W. From molecules to networks: adoption of systems approaches in circadian rhythm research. In: Andersen H, Dieks D, González W, Uebel T, Wheeler G, editors. New Challenges to Philosophy of Science. London, UK: Springer Verlag; 2013:211-223.

297. Li JZ. Circadian rhythms and mood: opportunities for multi-level analyses in genomics and neuroscience: circadian rhythm dysregulation in mood disorders provides clues to the brain's organizing principles, and a touchstone for genomics and neuroscience. BioEssays. 2014;36(3):305-315.

How to cite this article: McCarthy MJ, Gottlieb JF, Gonzalez

$\mathrm{R}$, et al. Neurobiological and behavioral mechanisms of circadian rhythm disruption in bipolar disorder: A critical multi-disciplinary literature review and agenda for future research from the ISBD task force on chronobiology. Bipolar Disord. 2021;00:1-32. doi:10.1111/bdi.13165 